\title{
ENHANCED VARIATIONAL IMAGE DEHAZING
}

\author{
ADRIAN GALDRAN* , JAVIER VAZQUEZ-CORRAL ${ }^{\dagger}$, DAVID PARDO I $^{\ddagger}$, AND \\ MARCELO BERTALMÍO ${ }^{\dagger}$
}

\begin{abstract}
Images obtained under adverse weather conditions, such as haze or fog, typically exhibit low contrast and faded colors, which may severely limit the visibility within the scene. Unveiling the image structure under the haze layer and recovering vivid colors out of a single image remains a challenging task, since the degradation is depth-dependent and conventional methods are unable to overcome this problem. In this work, we extend a well-known perception-inspired variational framework for single image dehazing. Two main improvements are proposed. First, we replace the value used by the framework for the grey-world hypothesis by an estimation of the mean of the clean image. Second, we add a set of new terms to the energy functional for maximizing the inter-channel contrast. Experimental results show that the proposed Enhanced Variational Image Dehazing (EVID) method outperforms other state-of-the-art methods both qualitatively and quantitatively. In particular, when the illuminant is uneven, our EVID method is the only one that recovers realistic colors, avoiding the appearance of strong chromatic artifacts.
\end{abstract}

Key words. image dehazing, perceptual color correction, contrast enhancement, variational image processing, visibility enhancement.

1. Introduction. Under good visibility conditions, the human eye can resolve two different objects only within a range of a few kilometers. Indeed, when looking at mid and long range open natural scenes, colors of far away parts of the scene tend to mix with the color of the atmosphere, gradually fading away and vanishing against the horizon. However, distance is not the only factor that can deteriorate perceptibility, but also bad weather conditions can accentuate visibility decrease. The presence of tiny suspended particles in the atmosphere lying between the observer and the objects in the scene can cause the deflection of light beams that travel from the latter to the former. This is produced by a wide range of factors, including dust, pollution, fog, haze, rain, or snow.

Recently, the problem of restoring and properly enhancing images affected by bad atmospheric conditions has attracted much attention in the image processing and computer vision community. From a practical point of view, many vision systems operating in real-world outdoor scenarios assume that the input is the unaltered scene radiance. These techniques designed for clear weather images may suffer under bad weather conditions where, even for the human eye, discerning image content can

*TECNALIA, Computer Vision Group. Parque Científico y Tecnológico, Edificio 700, E48160, Derio, Spain. E-mail: adrian.galdran@tecnalia.com.

$\dagger$ Department of Information and Communication Technologies, University Pompeu Fabra, Tànger, 122-140, E-08018, Barcelona, Spain.

J. Vazquez-Corral and M. Bertalmío were supported by European Research Council, Starting Grant ref. 306337, by the Spanish government, grant ref. TIN2012-38112, and by the Icrea Academia Award. E-mail: \{javier.vazquez, marcelo.bertalmio\}@upf.edu.

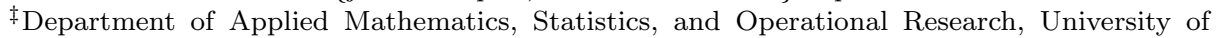
the Basque Country, Barrio Sarriena S/N, Campus de Leioa, E-48940, Leioa, Spain.

$\S$ BCAM, Basque Center for Applied Mathematics, Al. Mazarredo, 14, E-48009, Bilbao, Spain.

I Ikerbasque, Basque Foundation for Science, Al. Urquijo, 36-5, E-48011, Bilbao, Spain. D. Pardo was partially funded by the Project of the Spanish Ministry of Economy and Competitiveness with reference MTM2013-40824-P, the BCAM "Severo Ochoa" accreditation of excellence SEV-2013-0323, the CYTED 2011 project 712RT0449, and the Basque Government Consolidated Research Group Grant IT649-13 on "Mathematical Modeling, Simulation, and Industrial Applications (M2SI)”, Email:-dzubiaur@gmail.com. 


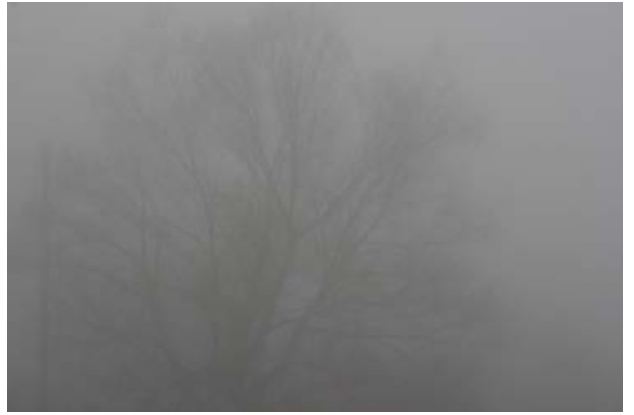

(a)

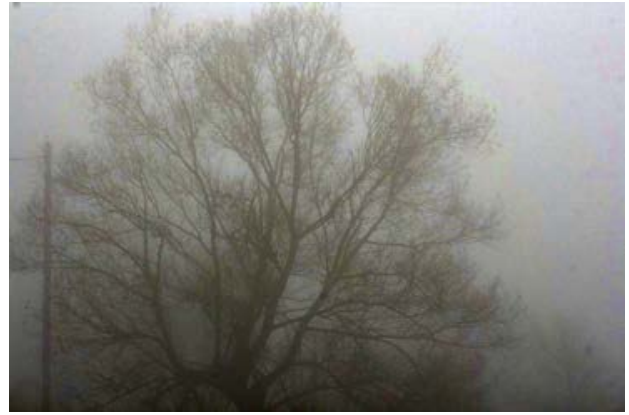

(b)

FIG. 1.1. (a) Hazy image, where the degradation increases with distance. A non-uniform illuminant dominates the scene. (b) The result of our dehazing method.

represent a serious challenge. Therefore, robustly recovering visual information in bad weather conditions is essential for several machine vision tasks, such as autonomous robot/vehicle navigation [15], remote sensing [22] or video surveillance systems [49, 40. Aerial and remotely sensed images, related to applications such as land cover classification [51, 23, and even underwater image restoration [6, 11] can also benefit from efficient dehazing techniques.

From a physical point of view, models of the propagation of light through the atmosphere can be traced back to the work of Kochsmieder [20, who formulated a simple linear model relating the luminance reflected by an object to the luminance reaching the observer. This model identified the distance between the observer and the source, as well as the particle composition of the atmosphere, as the key parameters that govern the decay of the luminance. From then on, studies of the interaction of light with the atmosphere continued growing as a rich field of research in the area of applied optics [27, 25].

Based on these physical models, many image dehazing methods have been proposed. They can be roughly classified into multiple image ones $33,29,39,30,31,32$, if they use more than one input image to estimate depth information, or single image ones [43, 17, 9], that work only with an initial degraded image. We can also find works that directly compute the albedo. In this case, a depth map can be obtained as a by-product of these methods. Examples of this approach include the work of Tan [42, where the albedo is estimated by imposing a local maximization of contrast, or Fattal 8, where it is assumed that depth and surface shading are locally uncorrelated. Unfortunately, both of these approaches rely on the assumption that depth is locally constant, and as a consequence, the obtained images may sometimes suffer from artifacts and tend to over-enhance the results.

This work is an extension of the conference work of [12. In this version, two main developments over the previous work have been introduced. First, we have extended the original functional, adding extra energy terms that allow us to control the degree of image saturation on the output. Second, we have conducted an exhaustive quantitative evaluation versus a large set of recent methods. As our experiments demonstrate, the minimization of our model provides high-quality haze-free images, even in challenging scenarios with uneven illumination or remarkably poor chromatic information. These seem to be situations where the majority of the state-of-the- 
art methods tend to encounter difficulties in restoring the degraded image without introducing color artifacts.

The rest of the paper is structured as follows. In the next section we review recent methods for image dehazing. Next, we formulate the image dehazing problem in a variational setting, and we develop our Enhanced Variational Image Dehazing (EVID) method. Section 4 is devoted to experimental results and comparison to other state-of-the-art methodologies. We end up in section 5 by summarizing our approach.

2. Related work. Most previous works on image dehazing are based on solving the image formation model presented by Koschmieder [20] that can be computed channel-wise as follows

$$
\mathrm{I}(x)=\mathrm{t}(x) \mathrm{J}(x)+(1-\mathrm{t}(x)) \mathrm{A},
$$

where $x$ is a pixel location, $\mathrm{I}(x)$ is the observed intensity, $\mathrm{J}(x)$ is the scene radiance, corresponding to the non-degraded image, transmission $\mathrm{t}(x)$ is a scalar quantity that is inversely related to the scene's depth, while A, known as airlight, plays the role of the haze color, which is usually considered constant over the scene, and therefore in a channel-wise formulation it is a scalar value.

Solving Eq. 2.1) is an under-constrained problem, i.e. there exist a large number of valid solutions. To constrain this indeterminacy, extra information in different forms has been introduced in the past. A large part of these works can be grouped under the category of methods that work with multiple input images or other data, which must be fused to take profit of this extra-information. For example, in [31], several instances of the same scene are acquired under different weather conditions. Then, differences in pixel intensities allow to estimate depth discontinuities, computing thus a scene structure map that enables the recovery of a clear weather scene. Schaul et al. acquire visible and near-infrared (NIR) images of the same scene [38, taking profit of the fact that NIR images are less sensitive to haze. Both images are then fused by means of a multiresolution scheme to obtain a dehazed image. The work in [19] retrieves depth information from geo-referenced digital urban and terrain models. An interactive registration of the photograph and its model is performed, after which depth information is available, making possible to remove haze effects in the input image. In [39, multiple images taken through a polarizer at different orientations are used. This approach relies on the fact that the light scattered by atmospheric particles is partially polarized to invert a physical model of image formation, enhancing scene contrast and correcting color. Unfortunately, all these methods depend on the acquisition or availability of extra information, which is often unavailable, and this hinders the practical use of these techniques.

Dehazing is particularly challenging when only a single input image is available. In this case, the majority of existing methods are also focused on solving Eq. (2.1) by inferring depth information. The method in [4] assumes that hazy images are usually acquired outdoors, allowing to conclude certain characteristics about the geometry of hazy scenarios. For example, the top part of the image is considered to contain the most hazy regions in the image. Tarel et al. propose in [43] a fast algorithm that estimates the atmospheric veil (equivalent to the depth map) through an optimization procedure in which they impose piecewise smoothness. The main advantage of this method is its low complexity, that they show to be a linear function of the number of pixels. More recently, several fusion-based dehazing strategies have also been proposed. In [1, a multi-scale fusion strategy is adopted, in which the authors compute several weighting maps (based on luminance, chromaticity and saliency), that they 
use to fuse a white-balanced and a contrast enhanced version of the original degraded image. In [48, the depth map is obtained by a fusion procedure in a probabilistic framework.

When inverting models like the one in Eq. (2.1), there is a risk of enhancing both the underlying signal and the noise. Some works have also studied this issue. For example, in [18, a variational formulation is proposed, in which a distance-dependent and edge-preserving regularization term is introduced, resulting in a numerical scheme that is able to dehaze the input image while denoising far away regions. The variational point of view has also been recently exploited in [7, where Fang et al. proposed a variational formulation intended to dehaze a degraded image while denoising it. After estimating the depth information using a variation of the dark channel prior, the authors formulated an image energy, that they minimized using the primal-dual Chambolle-Pock algorithm [5]. Existence of a minimizer and convergence of the numerical schemes were also discussed.

A special mention deserves the dark channel methodology [17, probably the most successful technique to date, due to its simplicity and effectiveness. This method is based on the statistical observation that haze-free images are colorful and contain textures and shadows, therefore lacking locally the presence of at least one of the three color components. On the contrary, hazy images present less contrast and saturation. As depth increases and the haze takes over the image, the contrast and saturation further decrease, providing an estimate of the depth information based on which it becomes possible to invert Eq. 2.1), obtaining high-quality results.

Several methods that are independent of an initial estimation of the scene depth have also been devised. Tan 42 ] observes that a haze-free image must have more contrast than a hazy one, and also that the amount of haze varies smoothly. Considering that nearby pixels tend to lie in the same depth layer of the scene, and thus are equally affected by haziness, he formulates a cost function attempting to maximize contrast while preserving depth smoothness locally. This cost function is optimized within a Markov random field framework, yielding a haze-free image. In [8], Fattal estimates scene depth by extending the image formation model to take into account both transmission and surface shading. He then looks for a haze-free solution of the model in which the resulting shading and transmission functions are locally uncorrelated in an statistical sense. In a subsequent work 9 , the same author builds on the concept of color-lines, establishing that for natural images, pixels exhibit a local one-dimensional distribution in the RGB space. A local image formation model is derived to adapt the color-lines concept to hazy images, and transmission in the scene is estimated by means of a Markov random field that also allows to control noise. A substantially different approach is adopted by Nishino et al. in 34. Rather than attempting to estimate depth in an initial stage, the authors resort to a Bayesian probabilistic framework, in which they are able to jointly estimate the albedo and the depth, by considering them as two statistically independent latent layers.

3. Enhanced Variational Image Dehazing (EVID) method. The majority of current dehazing algorithms are based on an estimation of the image depth (or transmission). Therefore, these methods are susceptible to fail when the physical assumptions underlying Eq. 2.1) are violated. This is a common phenomena both in real life, for example, when there is a source of light hidden by the haze, and in virtually-generated images that add different types of fog. Methods that do not estimate the model depth do not suffer from this problem, but they usually result in over-enhanced images due to the special characteristics of the degradation associated 
with haze. More conventional contrast enhancement algorithms, such as histogram equalization, are not suitable either. Fortunately, recent spatially-variant contrast enhancement techniques can be adapted to perform well for image dehazing tasks. In the following, we develop a variational framework for image dehazing that enforces contrast enhancement on hazy regions of the image throughout an iterative procedure allowing us to control the degree of restoration of the visibility in the scene.

3.1. Variational contrast enhancement. In 2007, Bertalmío et al. 3] presented a perceptually-inspired variational framework for contrast enhancement. Their method is based on the minimization of the following functional for each image channel I:

$$
E(\mathrm{I})=\frac{\alpha}{2} \sum_{x}\left(\mathrm{I}(x)-\frac{1}{2}\right)+\frac{\beta}{2} \sum_{x}\left(\mathrm{I}(x)-\mathrm{I}_{0}(x)\right)^{2}-\frac{\gamma}{2} \sum_{x, y} \omega(x, y)|\mathrm{I}(x)-\mathrm{I}(y)|,
$$

where $I$ is a color channel (red, green or blue) with values in $[0,1], I_{0}$ is the original image, $x, y$ are pixel coordinates, $\alpha, \beta, \gamma$ are positive parameters, and $\omega(x, y)$ is a positive distance function with its value decreasing as the distance between $x$ and $y$ increases. This method extends the idea of variational contrast enhancement presented by Sapiro and Caselles [37 and it also shows a close connection to the ACE method [36]. Bertalmío and co-authors have later revealed connections between this functional and the human visual system: they generalized it to better cope with perception results [35, and they established a very strong link with the Retinex theory of color [2].

The minimization of the image energy in Eq. (3.1) presents a competition between two positive terms and a negative one. The two positive terms prevent the solution from departing too much from the original image (second term) and preserve the grayworld hypothesis (first term). The negative competing term attempts to maximize the contrast. By focusing on this negative term of Eq. (3.1), we can observe a very useful relation with dehazing methods. It can be written as:

$$
\sum_{x, y} \omega(x, y)|\mathrm{I}(x)-\mathrm{I}(y)|=\sum_{x, y} \omega(x, y)(\max (\mathrm{I}(x), \mathrm{I}(y))-\min (\mathrm{I}(x), \mathrm{I}(y))) .
$$

We see from the above equation that the contrast term is maximized whenever the minimum decreases or the maximum increases, corresponding to a contrast stretching. Notice that minimization of local intensity values is one of the premises of a haze-free image, according to the Dark Channel prior [16. The second premise of this prior is the presence, locally, of a low intensity of Red, Green or Blue. We will extend our method to handle also this hypothesis is subsection 3.3 .

3.2. Modifying the Gray World assumption. In the image dehazing context, the Gray World hypothesis implemented in Eq. (3.1) is not adequate, since we want to respect the colors of the haze-free image, not to correct the illuminant of the scene. Different modifications of this hypothesis have already been proposed for several problems [10, 50. Here, to approximately predict which should be the mean value of a dehazed scene, we rely on the model of Eq. (2.1), that is written channel-wise as:

$$
\mathrm{I}^{j}=\mathrm{J}^{j} \mathrm{t}+(1-\mathrm{t}) A^{j},
$$

where $j \in\{R, G, B\}$. By rearranging and taking the average of each term, we can write:

$$
\operatorname{mean}\left(\mathrm{J}^{j} \cdot \mathrm{t}\right)=\operatorname{mean}\left(\mathrm{I}^{j}\right)-\operatorname{mean}\left((1-\mathrm{t}) A^{j}\right) .
$$


Now, we make the following two assumptions:

1. On a hazy image, color of a pixel is depth-dependent. On a haze-free image, however, we can expect colors to be independent of where the object is located in the scene. Thus, we can assume that $\mathrm{J}^{j}$ and $\mathrm{t}$ are uncorrelated, allowing us to write:

$$
\operatorname{mean}\left(\mathrm{J}^{j} \cdot \mathrm{t}\right)=\operatorname{mean}\left(\mathrm{J}^{j}\right) \cdot \operatorname{mean}(\mathrm{t})
$$

2. We assume also that $t$ has a uniform distribution across the image, i.e., depth values are equally distributed. This can be expressed simply as mean $(\mathrm{t})=$ $1 / 2$.

Using the above assumptions, we can estimate mean $\left(\mathrm{J}^{j}\right)$ as:

$$
\operatorname{mean}\left(\mathrm{J}^{j}\right) / 2 \approx \operatorname{mean}\left(\mathrm{I}^{j}\right)-(1 / 2) \operatorname{mean}\left(\mathrm{A}^{j}\right) .
$$

The airlight A takes a constant value for each channel that can be roughly approximated by the maximum intensity value on each component, since haze regions have usually higher intensity. Thus, a reasonable approximation for the mean value of the haze-free scene, and in consequence a new gray-world value, is given by:

$$
\mu^{j}=\operatorname{mean}\left(\mathrm{J}^{j}\right) \approx 2 \operatorname{mean}\left(\mathrm{I}^{j}\right)-\mathrm{A}^{j} .
$$

We can now rewrite the energy functional as:

$$
E\left(\mathrm{I}^{j}\right)=\frac{\alpha}{2} \sum_{x}\left(\mathrm{I}^{j}(x)-\mu^{j}\right)^{2}+\frac{\beta}{2} \sum_{x}\left(\mathrm{I}^{j}(x)-\mathrm{I}_{0}^{j}(x)\right)^{2}-\frac{\gamma}{2} \sum_{x, y} \omega(x, y)\left|\mathrm{I}^{j}(x)-\mathrm{I}^{j}(y)\right| .
$$

To minimize the above energy, we first need to compute its Euler-Lagrange derivative. In [3, the authors found that a minimizer of (3.5) must satisfy:

$$
\nabla E\left(\mathrm{I}^{j}\right)=\alpha\left(\mathrm{I}^{j}(x)-\mu_{j}\right)+\beta\left(\mathrm{I}^{j}(x)-\mathrm{I}_{0}^{j}(x)\right)-\gamma R\left(\mathrm{I}^{j}\right)(x)=0, \quad j \in\{R, G, B\}
$$

where the function $R(\mathrm{I})$ is a contrast enhancement operator:

$$
R(\mathrm{I})(x)=\frac{\sum_{y} \omega(x, y) s(\mathrm{I}(x)-\mathrm{I}(y))}{\sum_{y} \omega(x, y)},
$$

and $s$ is a smooth approximation of the sign function, that accounts for the first derivative of the absolute value. Details can be found in 3 . In the next subsection, we formulate an extended version of the energy given in (3.5). We also give in the Appendix a proof of the computation of its variational derivative. This proof generalizes the one included in [3].

We can now apply a gradient descent strategy. To this end, we solve $\frac{\delta \mathrm{I}}{\delta t}=$ $-\nabla E(\mathrm{I})$, being $t$ the evolution parameter. For the case of the energy given by Eq. (3.5), with the modified gray world assumption, after an explicit discretization in time, we have:

$$
\mathrm{I}_{k+1}^{j}=\mathrm{I}_{k}^{j}(1-\Delta t(\alpha+\beta))+\Delta t\left(\alpha \mu^{j}+\beta \mathrm{I}_{0}\right)+\Delta t \gamma R\left(\mathrm{I}_{k}^{j}\right), \quad j \in\{R, G, B\}
$$

The initial condition for this descent is $\mathrm{I}_{k=0}^{j}=\mathrm{I}^{j}(x)$. The computation of operator $R$ is reformulated in terms of convolutions by means of Fast Fourier Transforms. This brings a significant computational improvement to the method, since the effort to compute expression (3.7) falls down from $\mathcal{O}\left(N^{2}\right)$ to $\mathcal{O}(N \log (N))$. Details of this argument for complexity reduction can be found in [3, 13]. 
3.3. Controlling the saturation. The variational framework has several advantages over traditional closed-form solutions. First, it provides an iterative procedure that allows the user to stop the iterations as soon as the image meets a desired degree of quality. Also, the effect of the modification of the parameters governing the evolution of Eq. (3.8) is simple to understand and has a clear meaning. Furthermore, the energy-based formulation eases the combination of different image processing objectives. For example, we can combine Eq. 3.5 with a depth map coming from any of the algorithms that are able to estimate a 3D structure in the scene, to enforce denoising or deblurring tasks in far away areas of the scene. As an additional feature of the proposed method, in this section we illustrate how we can add an extra term to Eq. (3.5) allowing us to recover more vivid colors, in case the output image seems too washed-out, or if the user considers there is too much saturation in the recovered scene.

Hazy images are not only characterized by a loss of contrast, but they also exhibit a low saturation that makes colors look faint. The degree of saturation of a pixel $x$ in a RGB image is defined by the following formula:

$$
\begin{aligned}
\mathrm{S}(x) & =\frac{\max \left(\mathrm{I}^{R}(x), \mathrm{I}^{G}(x), \mathrm{I}^{B}(x)\right)-\min \left(\mathrm{I}^{R}(x), \mathrm{I}^{G}(x), \mathrm{I}^{B}(x)\right)}{\max \left(\mathrm{I}^{R}(x), \mathrm{I}^{G}(x), \mathrm{I}^{B}(x)\right)} \\
& =1-\frac{\min \left(\mathrm{I}^{R}(x), \mathrm{I}^{G}(x), \mathrm{I}^{B}(x)\right)}{\max \left(\mathrm{I}^{R}(x), \mathrm{I}^{G}(x), \mathrm{I}^{B}(x)\right)}
\end{aligned}
$$

This implies that saturation of a pixel is augmented as long as the maximum increases or the minimum decreases in the above formula. This idea complies in part with the Dark Channel prior, which states that in a local neighborhood around a haze-free pixel, we should find a low value of intensity in some of the $R G B$ channels. We have already included the spatial component of this assertion in Eq. 3.2 . The maximization of the expression there enforces locally the presence of low intensity pixels. To incorporate the chromatic component, we simply modify the contrast increase term given by Eq. 3.2 to account for the value of the pixel in the different channels:

$$
\sum_{x, y} \omega(x, y)\left|\mathrm{I}^{j}(x)-\mathrm{I}^{l}(y)\right|=\sum_{x, y} \omega(x, y)\left(\max \left(\mathrm{I}^{j}(x), \mathrm{I}^{l}(y)\right)-\min \left(\mathrm{I}^{j}(x), \mathrm{I}^{l}(y)\right)\right),
$$

where $j \neq l, j, l \in\{R, G, B\}$. This amounts to extending the energy in Eq. (3.5) by introducing an inter-channel stretching term:

$$
\begin{aligned}
E\left(\mathrm{I}^{j}\right)= & \frac{\alpha}{2} \sum_{x}\left(\mathrm{I}^{j}(x)-\mu^{j}\right)^{2}+\frac{\beta}{2} \sum_{x}\left(\mathrm{I}^{j}(x)-\mathrm{I}_{0}^{j}(x)\right)^{2}-\frac{\gamma}{2} \sum_{x, y} \omega(x, y)\left|\mathrm{I}^{j}(x)-\mathrm{I}^{j}(y)\right| \\
& -\left(\frac{\eta}{2} \sum_{x, y} \omega(x, y)\left|\mathrm{I}^{j}(x)-\mathrm{I}^{j+1}(y)\right|+\frac{\eta}{2} \sum_{x, y} \omega(x, y)\left|\mathrm{I}^{j}(x)-\mathrm{I}^{j+2}(y)\right|\right),
\end{aligned}
$$

where $j \in \mathbb{Z}_{3}$. In here, $\{R, G, B\}$ is identified with the space of integers modulo 3 , $\mathbb{Z}_{3}$, meaning for instance that $\mathrm{I}^{2}=\mathrm{I}^{B}$, and $\mathrm{I}^{3}=\mathrm{I}^{R}$. The derivative of this extended energy is given by the following expression:

$$
\begin{aligned}
\nabla E\left(\mathrm{I}^{j}\right)= & \alpha\left(\mathrm{I}^{j}(x)-\mu_{j}\right)+\beta\left(\mathrm{I}^{j}(x)-\mathrm{I}_{0}^{j}(x)\right) \\
& -\gamma R\left(\mathrm{I}^{j}, \mathrm{I}^{j}\right)(x)-\eta\left[R\left(\mathrm{I}^{j}, \mathrm{I}^{j+1}\right)+R\left(\mathrm{I}^{j}, \mathrm{I}^{j+2}\right)\right], \quad j \in \mathbb{Z}_{3},
\end{aligned}
$$




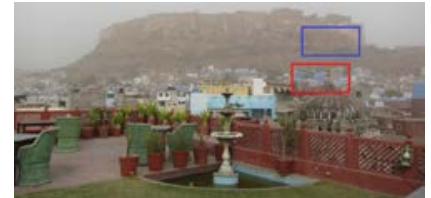

(a)

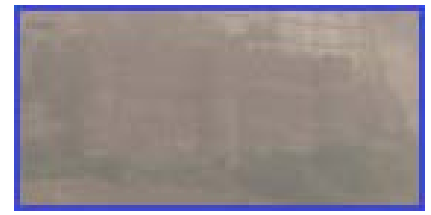

(d)

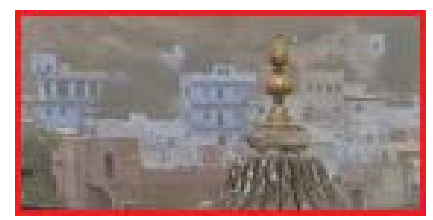

(g)

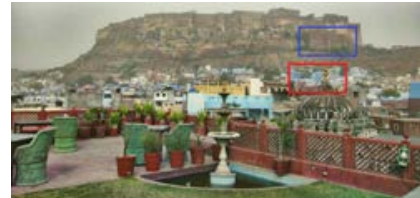

(b)

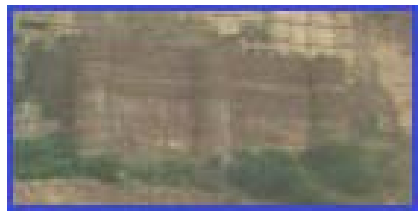

(e)

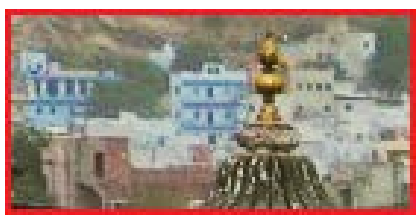

(h)

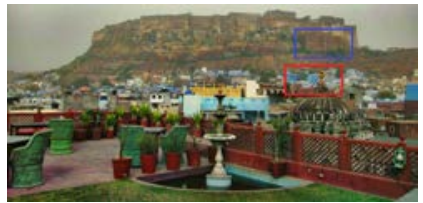

(c)

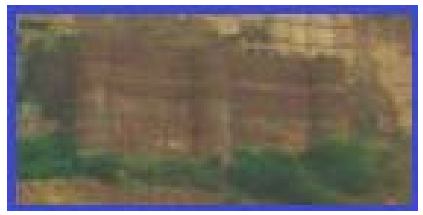

(f)

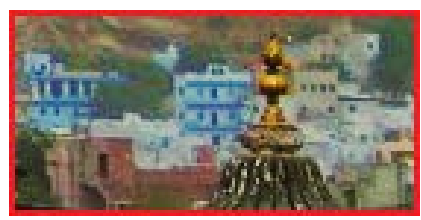

(i)

FIG. 3.1. (a) Hazy open scene. (b) Result of executing the method in Eq. (3.8). (c) Result of executing the method in Eq. 3.13). (d)-(f) Detail of the castle on top of the image (g)-(i) Detail of the buildings on the middle of the image.

where we have introduced an extension of the operator (3.7) by considering the operator $R\left(\mathrm{I}^{1}, \mathrm{I}^{2}\right)$ defined as follows:

$$
R\left(\mathrm{I}^{1}, \mathrm{I}^{2}\right)(x)=\frac{\sum_{y} \omega(x, y) s\left(\mathrm{I}^{1}(x)-\mathrm{I}^{2}(y)\right)}{\sum_{y} \omega(x, y)} .
$$

Notice that when $\mathrm{I}^{1}=\mathrm{I}^{2}$, this reduces to the mono-channel contrast enhancement operator defined in (3.7). Thus, the energy in Eq. 3.10 includes the one in Eq. 3.5 as a particular case, and so does its derivative. We include a proof of equation (3.11) in the appendix.

Now, a complete numerical gradient descent that increases local contrast and saturation can be written as:

$$
\begin{aligned}
\mathrm{I}_{k+1}^{j}= & \mathrm{I}_{k}^{j}(1-\Delta t(\alpha+\beta))+\Delta t\left(\alpha \mu^{j}+\beta \mathrm{I}_{0}\right) \\
& +\Delta t\left(\gamma R\left(\mathrm{I}_{k}^{j}, \mathrm{I}_{k}^{j}\right)+\eta\left[R\left(\mathrm{I}_{k}^{j}, \mathrm{I}_{k}^{j+1}\right)+R\left(\mathrm{I}_{k}^{j}, \mathrm{I}_{k}^{j+2}\right)\right]\right),
\end{aligned}
$$

where $j \in \mathbb{Z}_{3}$. Parameter $\eta$ controls the degree of saturation increase. If necessary, a change of sign in the last term of (3.13) would accomplish a local saturation decrease.

The iteration of Eq. 3.13 until steady-state configures our proposed Enhanced Variational Image Dehazing (EVID) method. EVID provides the user with a mechanism to control the amount of saturation on the recovered colors. As an example, in Fig. 3.1a we display a hazy open scene. The enhanced versions of the input image appearing in Fig. (3.1a) have been obtained by using Eqs. (3.8) and the EVID method, respectively. The convenience of applying EVID over the method of Eq. 
(3.8) is demonstrated in the close-up details appearing in Figs. (3.1d) to (3.1i). We can appreciate how the addition of the inter-channel saturation term produces more intense colors both in far away regions of the scene and in middle-range areas.

4. Experimental results. In this section, the dehazing capability of our EVID method is tested qualitatively and quantitatively by comparing it with several existing techniques. To qualitatively evaluate our method, we have executed it over various hazy images that are quite popular in the literature of image dehazing for evaluation purposes, as well as in other realistic scenarios, in which the illumination affecting the scene is non-uniform and the physical model in Eq. 2.1 becomes invalid. Likewise, for the quantitative evaluation of the experimental results, we make use of real depth data provided by 41 to produce both homogeneous and heterogeneous haze layers over real-world scenarios. We then compare the ability of our method to remove that haze layer by means of several standard full-reference metrics, comparing also against various state-of-the-art approaches.

Regarding the parameter configuration, we have considered Eq. 3.13 with the following baseline values: $\alpha=0.5, \beta=0.5, \gamma=0.2$, and $\eta=0.02$. Both distance functions were defined as Gaussians with kernel of standard deviation equal to 50 pixels. The time step was set to $\Delta t=0.15$, and we considered that a steady-state of the gradient descent was achieved when the difference between the images of two consecutive iterations was below 0.020 . A sensitivity study with respect to the parameters of our methods is provided in Section 4.1.3.

4.1. Qualitative evaluation. To evaluate the performance of a dehazing technique, the most popular criteria are often the amount of retrieved visibility for far away details, together with the plausibility of the colors that appear in the recovered scene. In this section, we consider also these subjective measurements to comment on the performance of our method when compared with other existing approaches.

4.1.1. Comparisons to state-of-the-art methods. Fig. 4.1 displays a hazy scene typically used as a benchmark for testing a dehazing method. In this case, all methods effectively recover visibility of hidden details at the bottom of the scene, being the technique of Meng et al. [26] the one that retrieves slightly more structure than the rest. Unfortunately, this seems to happen at the price of introducing some color artifacts at the leftmost part of the image. The rest of the methods are free from these artifacts.

On the other hand, the image in Fig. $4.1 \mathrm{a}$ is a particularly interesting test case, since the headlights of the train are useful to evaluate the way each method handles other light sources different from the ambient light. We can appreciate how only our EVID method and the one of Tarel et al. 43] are capable of correctly preserving the shape of the three lights. However, in this case our method recovers more vivid colors than [43, as can be seen by observation of the red wagon at the left of Figs. 4.1c and 4.1f).

Figure 4.2 displays an image of New York City, also often considered for benchmarking of dehazing algorithms. Therein, our EVID method is compared against the works in $[8,42,19,9,17,43$. Visibility of distant objects is again recovered by every method up to a reasonable degree. The recent method of Fattal [9] and the Dark Channel method [17] are possibly the ones recovering most realistic colors, although a close analysis reveals that they fail to recover the information underlying in the horizon (see upper right corner in Figs. (4.1e) and (4.1f)). Also, the method by Tan 42] suffers of noticeable over-saturation artifacts. 


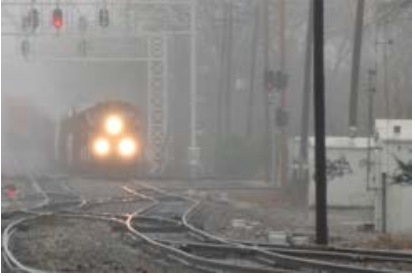

(a)

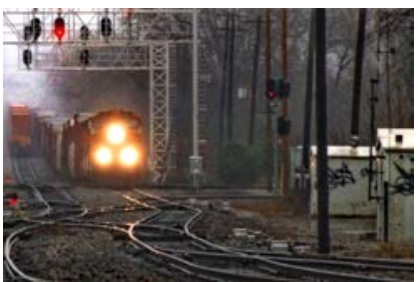

(d)

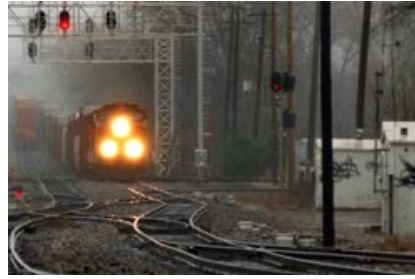

(b)

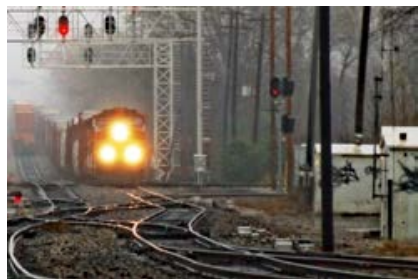

(e)

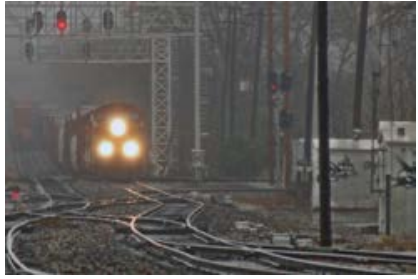

(c)

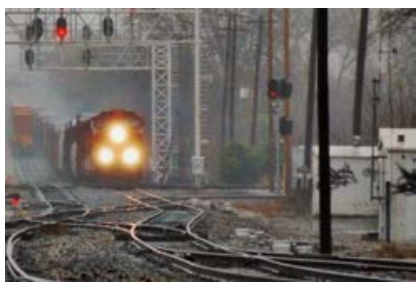

(f)

FIG. 4.1. (a) Typical benchmark image for dehazing algorithms. (b) to (f): Result of processing with the following methods: (b) He et al. [17]. (c) Tarel et al. [43]. (d)Meng et al. [26]. (e)Gibson et al. [14]. (f) Our proposed EVID method.

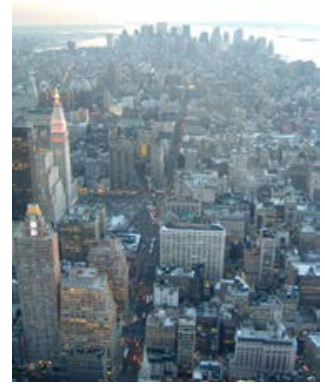

(a)

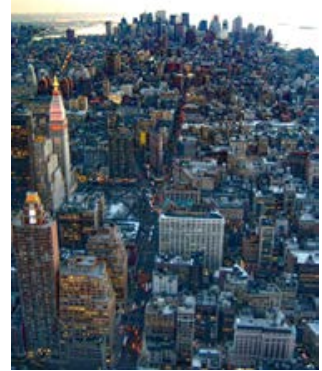

(e)

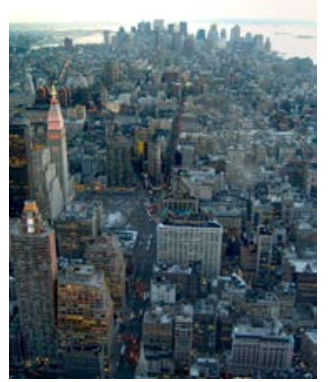

(b)

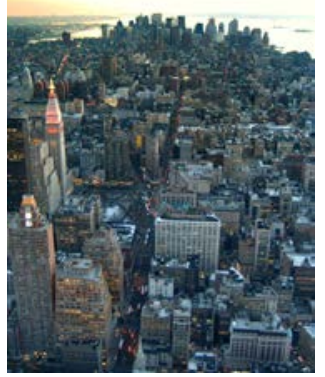

(f)

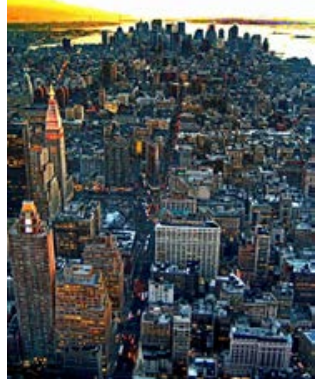

(c)

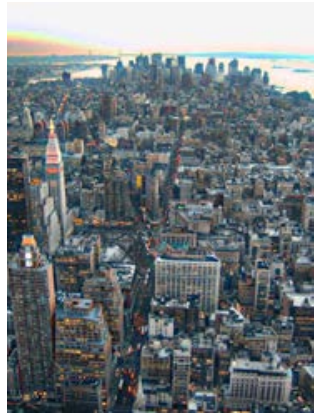

(g)

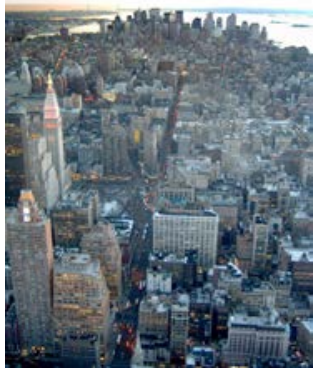

(d)

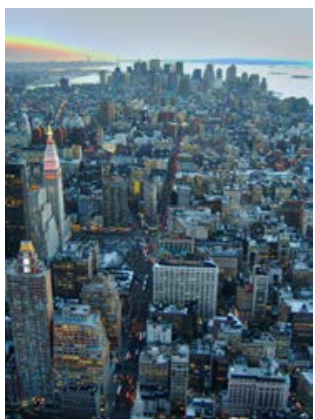

(h)

FIG. 4.2. (a) New York City image (input).Result of processing with methods of: (b) Fattal [8] (c) Tan. [42] (d) Kopf et al. [19] (e) Fattal [9] (f) He et al. [17] (g) Tarel et al. [43] (h) Our proposed EVID method. 
The previous two examples demonstrate how for typical images, existing algorithms (including ours) can handle haze effectively, recovering visibility up to some extent. On the other hand, each of the methodologies restores chromatic information in a different way, although the majority of the available techniques produce rather plausible colors. Differences in the performance are subtle and only little details reveal whether a method is performing better than another in particular regions of the image.

Unfortunately, little research has addressed the problem of image dehazing in a more challenging and also realistic scenario, such as the one depicted in Fig. 4.3 or Fig. 4.4. Let us notice that most of the state-of-the-art methodologies rely on the previous computation of a depth map of the scene. They usually resort to a physical model of the image formation under haze and bad weather conditions, such as Eq. 2.1. This model assumes constant illumination in the scene. When this assumption is violated, the airlight cannot be considered to be constant. The result is a transmission underestimate or overestimate in unevenly illuminated areas, and color distortions characterized by dark blue regions appear in the restored images.

The method proposed in this work benefits from the advantage of depending only mildly on physical considerations. The EVID method does not need to compute any depth information prior to restoration. Thanks to this feature, unevenly illuminated regions are handled properly, and scene structure can be recovered without introducing excessive color distortion. Fig. 4.3 clearly demonstrates this point. The original image in Fig. 4.3a was extracted from [48. The sun appears behind the haze at the upper left part of the image, producing a non-uniform illuminant: the leftmost part of the sky appears brighter than the rightmost side. The methods that rely on an accurate estimate of the airlight and the depth are fooled by this non-uniformity, and generate strong color artifacts. In this case, only our method is capable of retrieving some of the buildings structure without producing a chromatic degradation.

The same challenging scenario is faced in Fig. 4.4. Again, the compared methods cannot avoid the creation of color distortions in the effort to increase contrast in the upper rightmost part of the original hazy image. In this case, the EVID method was executed with a single parameter modification: $\gamma$ was set to 1 , forcing the solution to stay closer to the original image. This represents another advantageous feature of the variational approach, since the attachment to data term provides the user with an intuitive way of controlling whether the contrast increase is excessive or insufficient.

The example in Fig. 4.5 shows another useful characteristic of our approach. Most works rely on the presence of enough color information in the scene so as to recover the depth structure. When this chromatic information is weak or missing, the result is often also an image with unpleasant color artifacts. The EVID method operates in a channel-wise manner, handling thus more robustly the lack of color cues in the input image, as can be observed in the results obtained by the other physicallybased techniques when compared to our variational approach, see Fig. 4.5f. As in the previous image, a single parameter variation was considered for this image: $\gamma$ was set to 1.5, forcing the solution to stay closer to the original image.

Fig. 4.6 presents another image suffering from poor chromatic information. As in the previous case, our method seems to be the only one capable of restoring content in far away areas of the scene without introducing a remarkable color degradation.

4.1.2. Comparisons with contrast enhancement approaches. The results described in the previous section clearly illustrate the advantages of the EVID method over other recent dehazing techniques. Most of these advantages come from the fact 


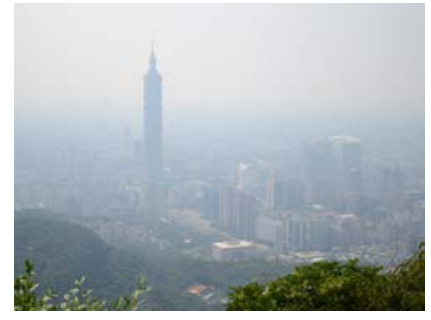

(a)

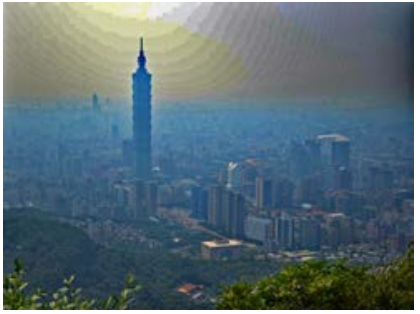

(d)

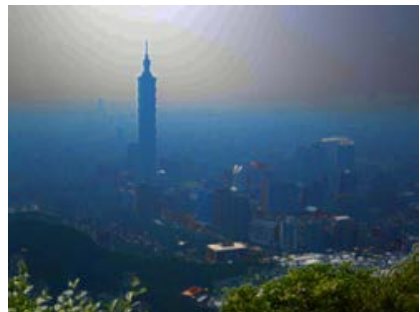

(b)

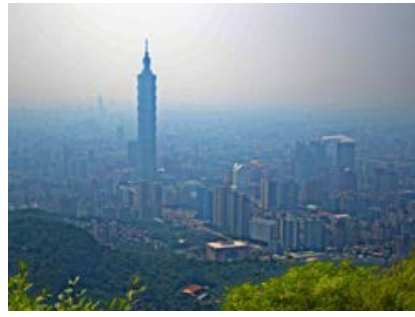

(e)

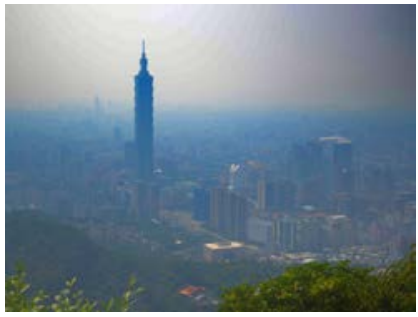

(c)

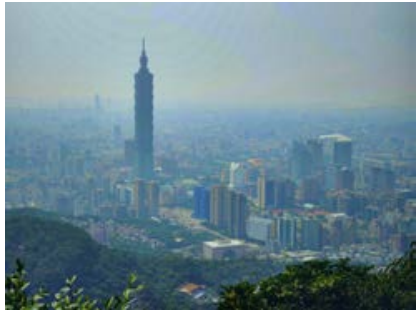

(f)

FIG. 4.3. (a) Unevenly illuminated hazy image extracted from [48]. Result of processing with methods of (b) Wang et al. method [48]. (c) He et al. [17]. (d) Meng et al. [26]. (e) Nishino et al. method [34]. (f) Our proposed EVID method.
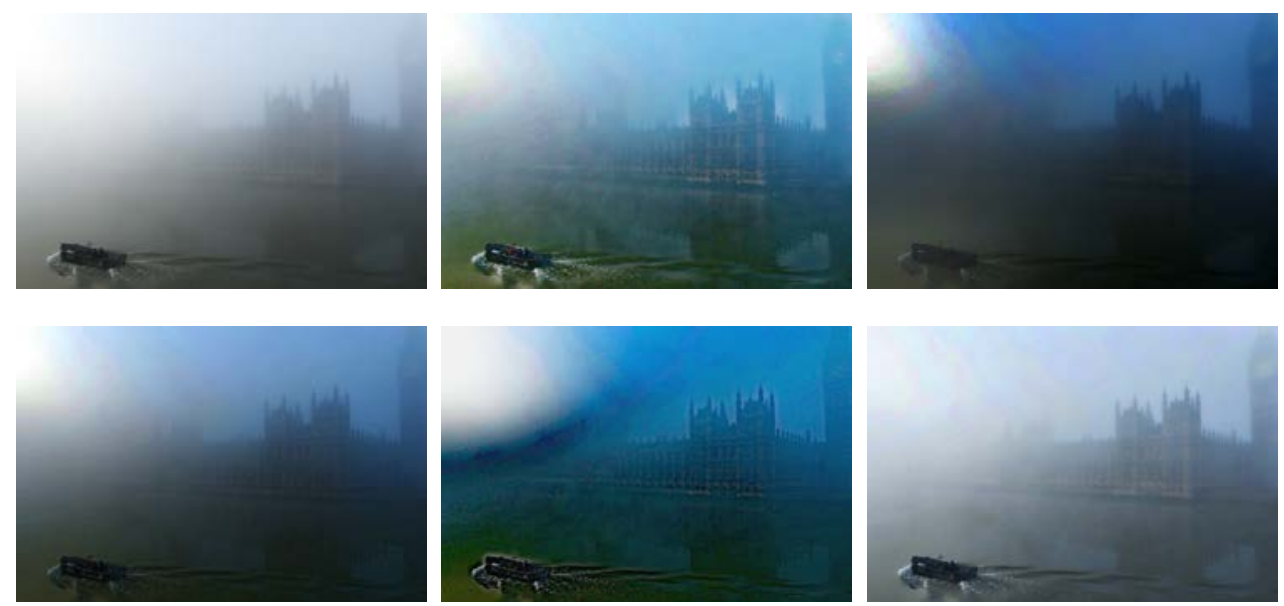

FIG. 4.4. a) Unevenly illuminated hazy image of the Thames river Result of processing with methods of b) Tarel et al. [43] c) He et al. [17] d) Gibson et al. '13 [14] e) Nishino et al. '12 [34] f) Our proposed EVID method.

that EVID does not compute a transmission map of the hazy scene. On the other hand, we do incorporate in the model a mild physical constraint, since our modified Gray-World hypothesis relies on an estimate of the mean of the haze-free scene, and this is obtained from the physical model given by Eq. (2.1). This also explains the improvement of EVID with respect to other contrast enhancement methods that do not consider any physical cue. To illustrate this point, we show in Fig. 4.7 the result 


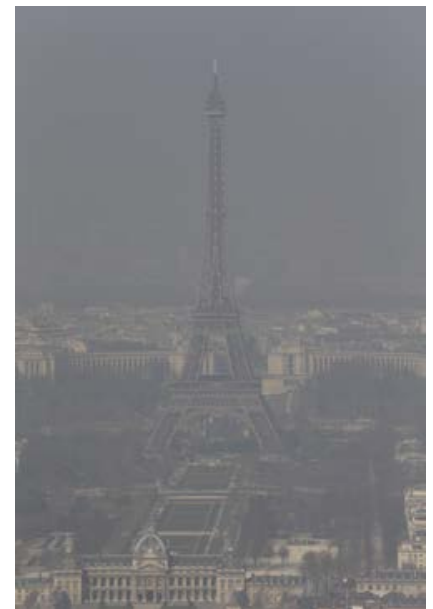

(a)

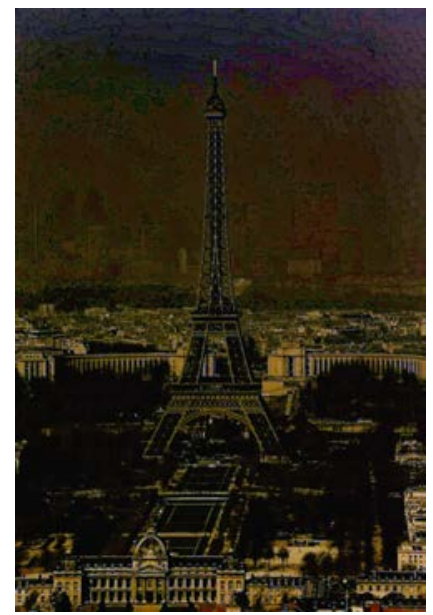

(d)

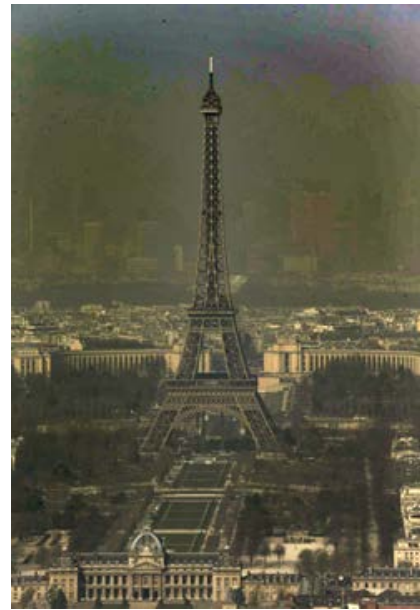

(b)

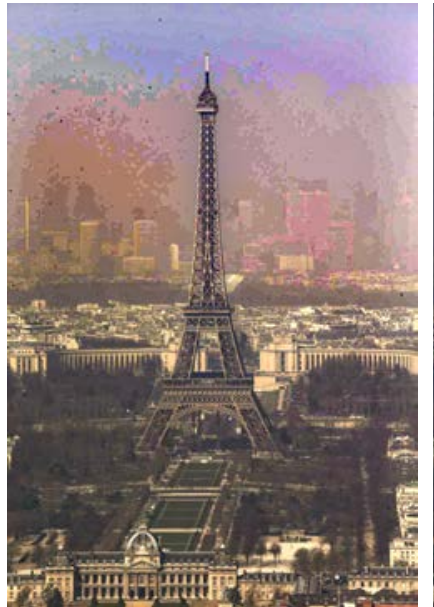

(e)

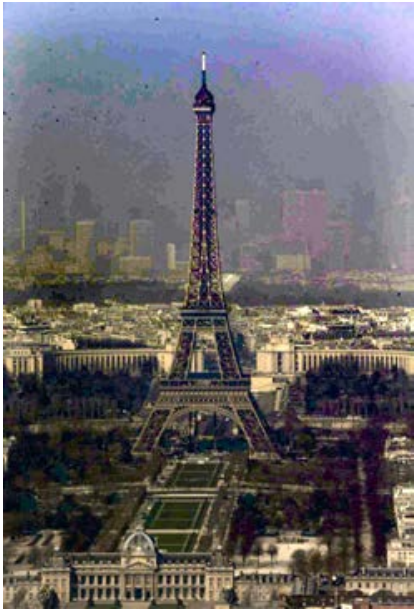

(c)

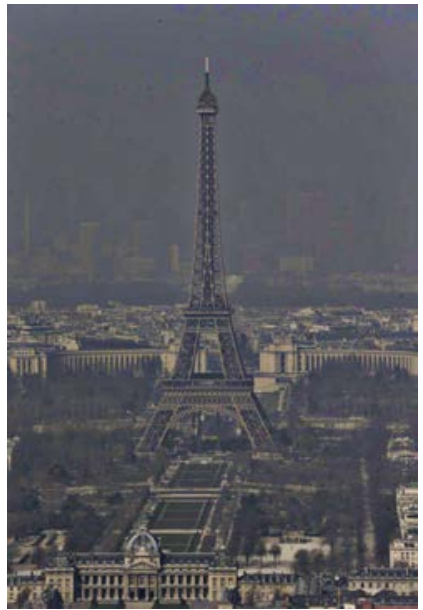

(f)

FIG. 4.5. (a) An image of Paris, with a lack of chromatic information due to haze in the scene. Result of processing with methods of (b) He et al. [17]. (c) Fattal [9]. (d) Nishino et al. [34]. (e) Meng et al. [26] (f) Our proposed method.

of processing three hazy scenes with EVID, as well as with a conventional contrast enhancement method (Histogram Equalization, HE) and the advanced variational contrast enhancement model on which the EVID method builds, introduced in 3 and described by Eq. (3.1). Notice that for a fair comparison, this model is run here with a parameter configuration analogous to the one of EVID.

In the three examples shown, we can clearly appreciate how EVID produces more vivid colors while increasing contrast. In Fig. 4.7a we see the same hazy image of a train as in Fig. 4.1a). HE saturates large parts of the image, in an attempt to obtain a global uniform histogram. On the other hand, the method in 3 does not saturate bright areas, but it seems unable to retrieve plausible colors in far-away regions of the scene, such as the red wagon or the green bush next to the train. 


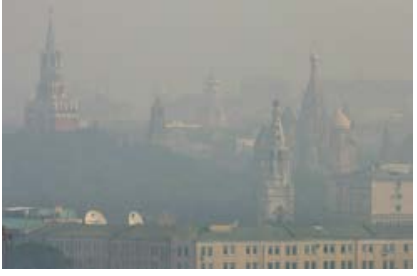

(a)

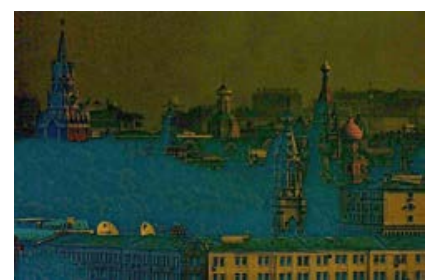

(d)

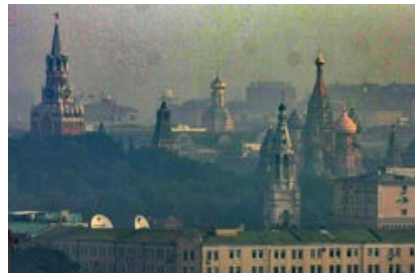

(b)

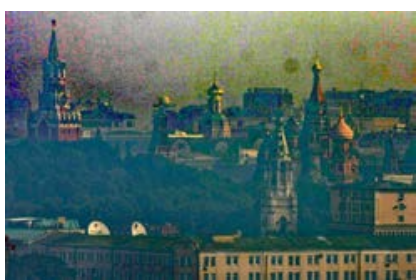

(e)

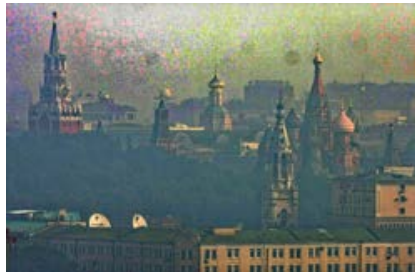

(c)

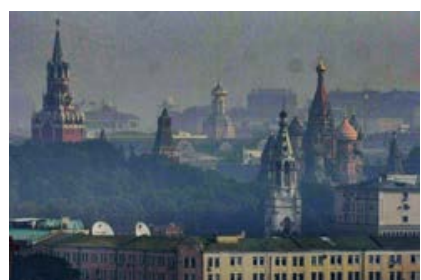

(f)

FIG. 4.6. (a) An image of Moscow, with a lack of chromatic information due to haze in the scene. Result of processing with methods of (b) He et al. [17]. (c) Meng et al. [26]. (d) Nishino et al. [34]. (e) Fattal [9]. (f) Our proposed method.

In Fig. 4.7e, we have a natural landscape with a forest on it. Again, HE burns dark regions while saturating to white on brighter areas. However, in this case, the method in [3] is capable of retrieving a similar contrast when compared to EVID. Unfortunately, due to the Gray World hypothesis implemented by [3], the model lacks the ability to produce intense colors.

Finally, in Fig. (4.7i) we include a hazy view of the city of Taipei. We can appreciate similar drawbacks for HE, which forces dark areas to become black and handles incorrectly the sky areas, saturating to white the top part of the image. Also, the method in [3] recovers some contrast, but again it produces grayish colors due to the Gray World assumption it incorporates, further supporting the idea that a smart contrast enhancement is not enough to approach the problem of haze removal. In this case too, the EVID method is able to recover contrast while producing richer colors. All these observations can be better appreciated in Fig. 4.8, where we have included close-up details of the image areas marked by blue, red, and yellow rectangles on Figs. 4.7a), 4.7e, and 4.7i).

4.1.3. Parameter Sensitivity. In this section, we study the influence of the different parameters presented in the model (degree of contrast, attachment to initial input, and saturation enhancement).

Let us first analyse the influence of the parameter $\gamma$, which controls the degree of contrast enhancement provided by the method. Fig. 4.9a shows a hazy scene of the city of Bilbao. The restored version displayed in Fig. (4.9c) has been obtained with the configuration of parameters recommended above, namely $\alpha=0.5, \beta=0.5$, $\gamma=0.2$, and $\eta=0.02$. The images appearing in Figs. $4.9 \mathrm{~b}$ and $4.9 \mathrm{~d})$ have been produced by varying parameter $\gamma$. Specifically, we have set $\gamma=0.1$ and $\gamma=0.3$. We can appreciate how this modification produces a smooth variation of contrast in the scene, with an insufficient increase in contrast for $\gamma=0.1$, and a slightly overenhanced output for $\gamma=0.3$. This can better appreciated in the close-up details of 


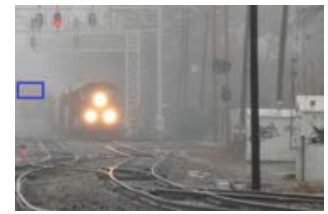

(a)

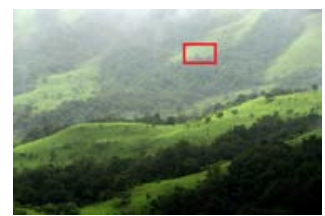

(e)

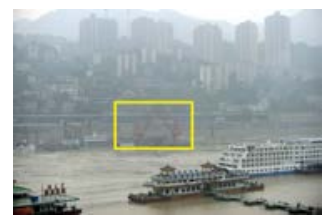

(i)

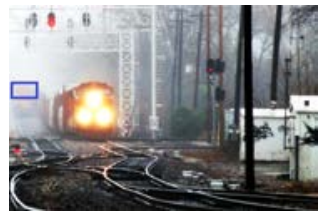

(b)

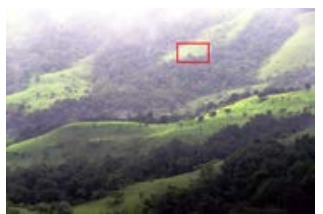

(f)

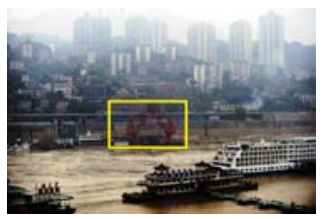

(j)

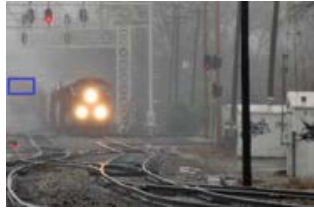

(c)

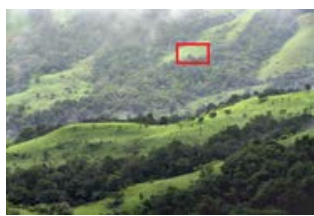

(g)

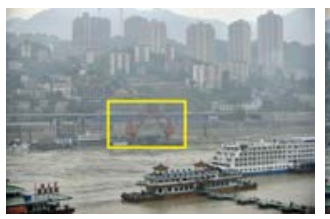

(k)

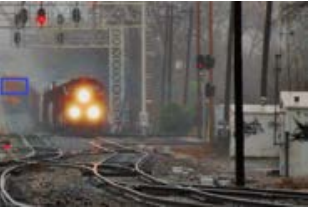

(d)

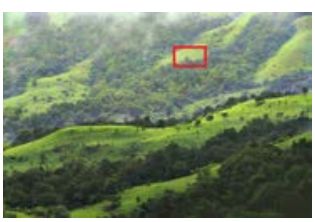

(h)

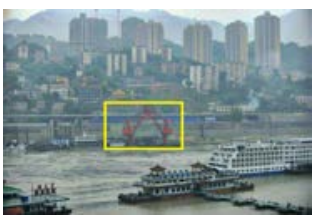

(1)

FIG. 4.7. Result of applying a conventional and an advanced contrast enhancement method compared with the result obtained by EVID. (a), (e), (i) Different hazy scenes. (b), (f), (j) Result of applying Histogram Equalization. (c), (g), (k) Result of applying the method in [3]. (d), ( $h$ ), (l) Our proposed EVID method.

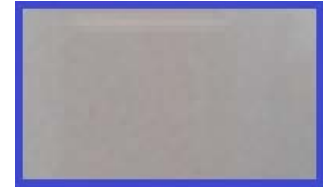

(a)

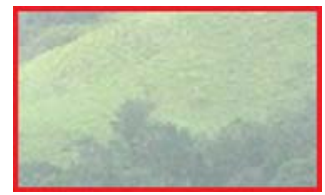

(e)

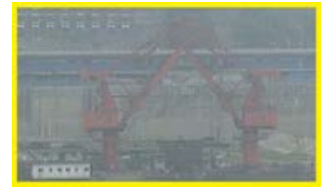

(i)

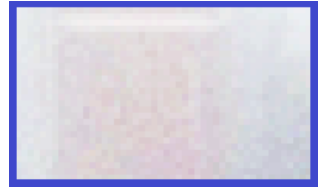

(b)

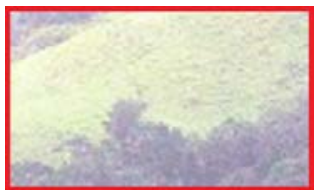

(f)

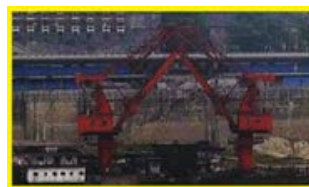

(j)

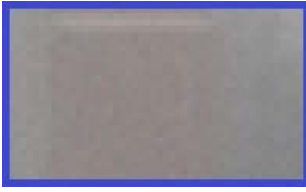

(c)

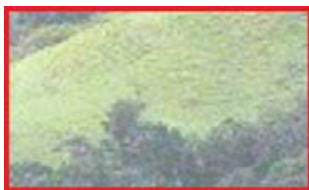

(g)

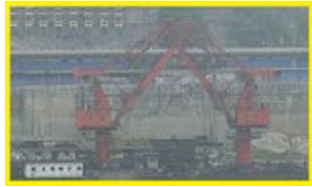

$(\mathrm{k})$

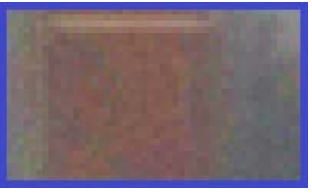

(d)

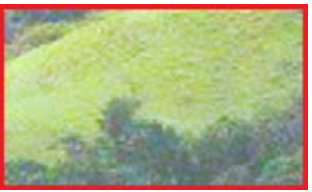

(h)

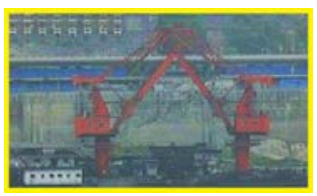

(1)

FIG. 4.8. Zoomed details from Fig. 4.7). (a)-(d) Detail of the red wagon in the left of the image for the original image, and the output produced by HE, [3], and EVID. (e) - (f) Detail of the trees in the top part of the scene for the original image, and the output produced by HE, [3], and EVID. (i)-(l)Detail of the crane in the middle of the scene for the original image, and the output produced by $H E$, [3], and EVID. 


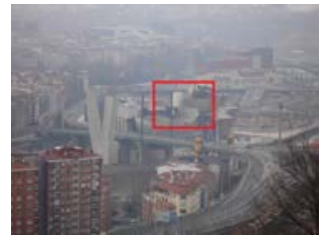

(a)

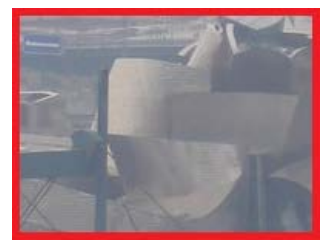

(e)

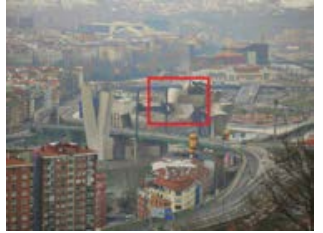

(b)

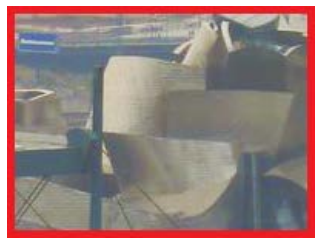

(f)

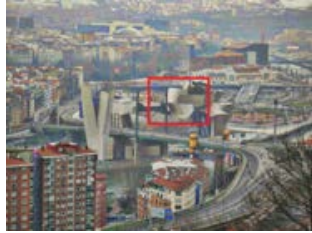

(c)

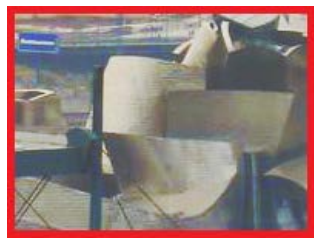

(g)

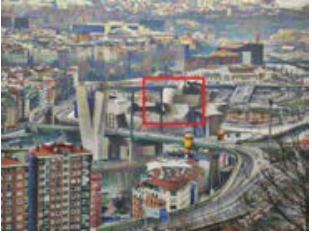

(d)

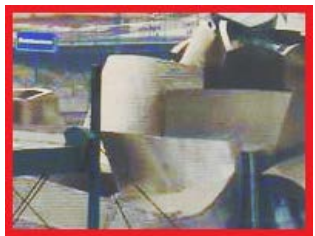

(h)

FIG. 4.9. A hazy view of the city of Bilbao, and the result of varying contrast parameter $\gamma$. (a) Original hazy scene. (b) $\gamma=0.1$ (c) $\gamma=0.2$ (d) $\gamma=0.3$. (e)-(h) Detail of the Guggenheim museum facade.

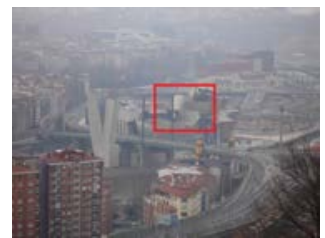

(a)

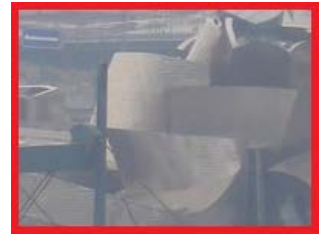

(e)

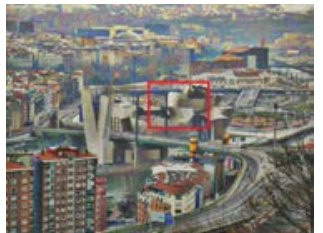

(b)

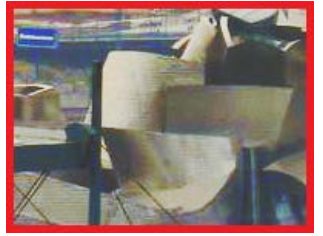

(f)

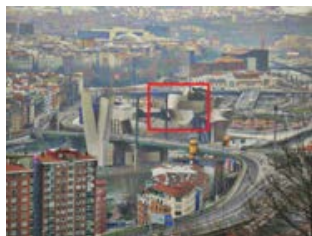

(c)

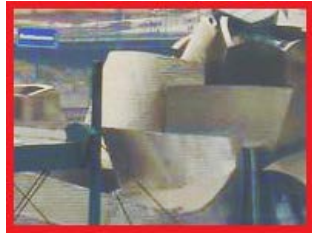

(g)

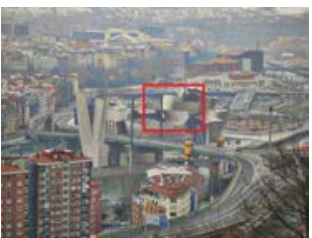

(d)

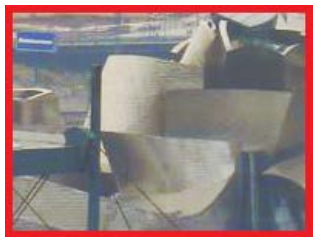

(h)

FIG. 4.10. A hazy view of the city of Bilbao, and the result of varying attachment-to-data parameter $\beta$. (a) Original hazy scene. (b) $\beta=0.25$ (c) $\beta=0.5$ (d) $\beta=0.75$. (e)-(h) Detail of the Guggenheim museum facade.

the museum's facade displayed on Figs. 4.9e to 4.9h.

To assess the effect of a varying attachment-to-data parameter, we have executed again EVID on the hazy image 4.9a), varying $\beta$ in the set $\{0.25,0.5,0.75\}$ and keeping fixed the other parameters on its baseline configuration, i.e. $\gamma=0.2, \beta=0.5$ and $\eta=$ 0.02 . The effect produced by increasing parameter $\beta$ is opposite to the one produced by increasing the contrast parameter $\gamma$, as expected. We can observe the result of this on Fig. 4.10. There, we can appreciate how the variation of the attachment-to-data parameter influences the degree of separation of the output image from the original one, allowing the user to control the degree of enhancement provided by the method.

The gray-world parameter captures the degree of confidence we have on the esti- 


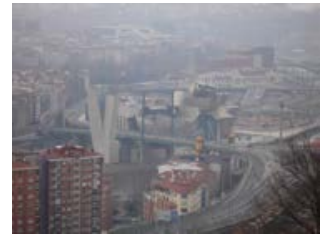

(a)

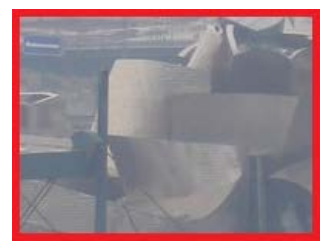

(e)

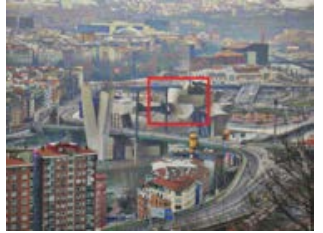

(b)

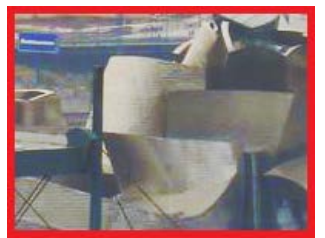

(f)

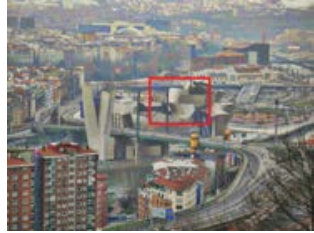

(c)

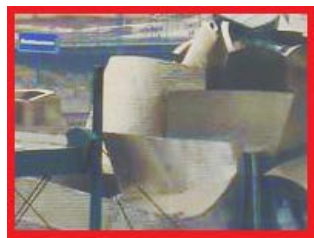

(g)

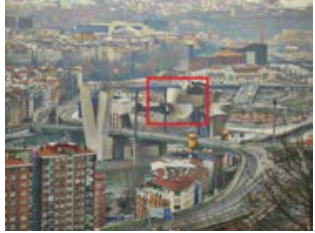

(d)

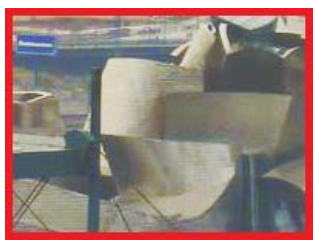

(h)

FIG. 4.11. A hazy view of the city Bilbao, and the result of varying gray-world parameter $\beta$. (a) Original hazy scene. (b) $\alpha=0.25$ (c) $\alpha=0.5$ (d) $\alpha=0.75$. (e)-(h) Detail of the Guggenheim museum facade.

mate of the mean of the haze-free image in accordance to Kochsmieder model. The effect of varying this parameter can be appreciated on Fig. (4.11), where we have executed the EVID method with the parameter reference configuration, and with $\alpha \in\{0.25,0.5,0.75\}$. A slight color shift can be noted in the different outputs. This is a global effect that can be better appreciated with a close look into the high resolution versions of the processed images.

Finally, the effect of modifying parameter $\eta$ affects to the amount of saturation that colors in the scene experiment. This is illustrated on Fig. 4.12, where he have executed the EVID method with the baseline parameter configuration, and setting $\eta \in\{0.01,0.02,0.03\}$. Color saturation gradually grows as $\eta$ increases. We can also appreciate in the far away buildings and trees the effect produced by a large value of $\eta$, leading to the appearance of color artifacts on large uniform areas.

4.2. Quantitative Evaluation. Evaluating the performance of a dehazing method is always a hard task due to the inherent lack of ground-truth. To overcome this difficulty, some previous works have resorted to the generation of a synthetic fog layer [45, 44, 21]. However, to be able to generate a realistic haze, we first need depth data on the scene. To this end, we employ in this work the LIVE Color+3D Database provided in [41. This database includes twelve different scenes together with real depth data. Data was acquired using an advanced range scanner with a digital camera mounted on top of it.

To incorporate realistic fog layers on top of these images, we employed the approach of Tarel et al. [44. The authors provide software to generate up to four different kinds of fog. This is achieved by slightly modifying Eq. (2.1) to introduce some variability, adding Perlin's noise to both transmission t and airlight A. The only parameter required by the method was the visibility distance, which was set to 60 meters for all the experiments. An example of an original image in the LIVE Color+3D Database, together with its ground-truth depth data, and the different hazy versions of the input image can be seen in Fig. 4.13).

We have therefore a total of 48 images (12 scenes under 4 different fogs). We 


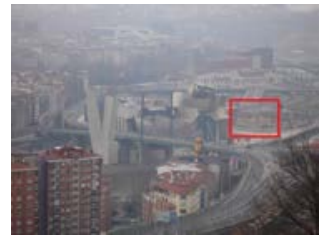

(a)

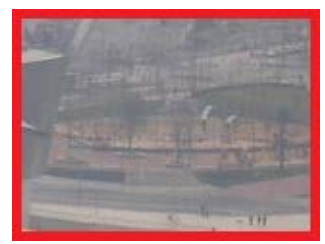

(e)

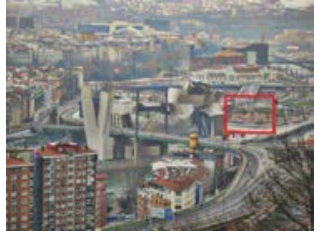

(b)

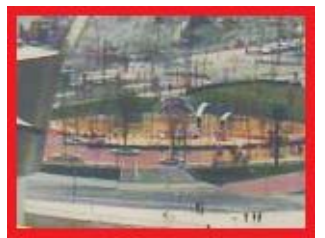

(f)

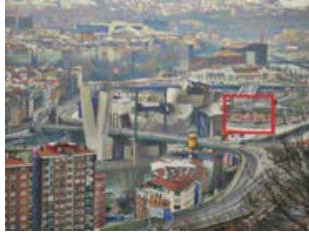

(c)

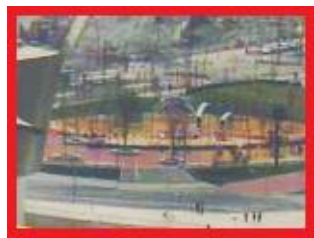

(g)

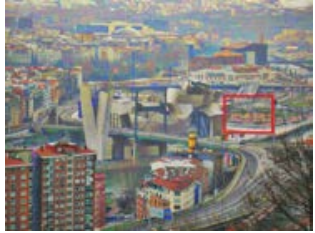

(d)

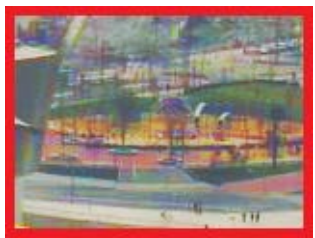

(h)

FIG. 4.12. A hazy view of the city of Bilbao, and the result of varying saturation parameter $\eta$. (a) Original hazy scene. (b) $\eta=0$ (c) $\eta=0.02$ (d) $\eta=0.04$. (e)-(h) Detail of the park next to the museum.

have compared EVID versus current state-of-the-art methods, namely the ones of $\mathrm{He}$ et al. [17, Meng et al. 26, Nishino et al. 34, Tarel and Hautière [43, and Gibson and Nguyen [14]. Our method was computed with the same fixed parameters for all scenes, as pointed out at the beginning of section 4 .

We have used five different measures, three mainly based on the Mean Square Error (MSE), and two mainly based on the correlation. The first considered MSE based measure consists of the computation of the MSE between the images for each particular channel, and then, the combination of the results of the three channels by the $l_{2}$ norm, numerically

$$
M S E_{\text {split }}=\sqrt{M S E\left(I_{r}^{G T}, I_{r}^{m}\right)^{2}+M S E\left(I_{g}^{G T}, I_{g}^{m}\right)^{2}+M S E\left(I_{b}^{G T}, I_{b}^{m}\right)^{2}},
$$

where $I^{G T}$ stands for the ground-truth image, $I^{m}$ stands for the method used, the subindexes represent each color channel and

$$
\operatorname{MSE}\left(I^{1}, I^{2}\right)=\frac{\sum_{i=1}^{M}\left(I^{1}(i)-I^{2}(i)\right)^{2}}{M},
$$

being $M$ the number of pixels.

The second error measure is the MSE between the luminance of the two images. Let us define $I_{\text {lum }}^{G T}=0.2126 I_{r}^{G T}+0.7152 I_{g}^{G T}+0.0722 I_{b}^{G T}$ and $I_{\text {lum }}^{m}=0.2126 I_{r}^{m}+$ $0.7152 I_{g}^{m}+0.0722 I_{b}^{m}$. Therefore, the error measure becomes:

$$
M S E_{\text {lum }}=M S E\left(I_{\text {lum }}^{G T}, I_{\text {lum }}^{m}\right) .
$$

We have also defined a new error measure in order to also account for the relation between the three color channels. We denote this error measure as $l_{2}-$ color and we define it as

$$
l_{2}-\text { color }=\frac{\sum_{i=1}^{M} \sqrt{\left(I_{r}^{G T}(i)-I_{r}^{m}(i)\right)^{2}+\left(I_{g}^{G T}(i)-I_{g}^{m}(i)\right)^{2}+\left(I_{b}^{G T}(i)-I_{b}^{m}(i)\right)^{2}}}{M} .
$$




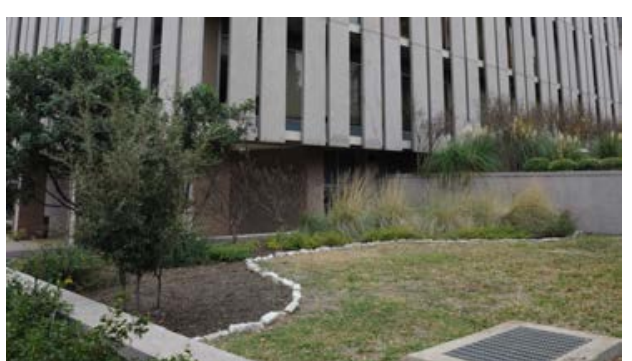

(a)

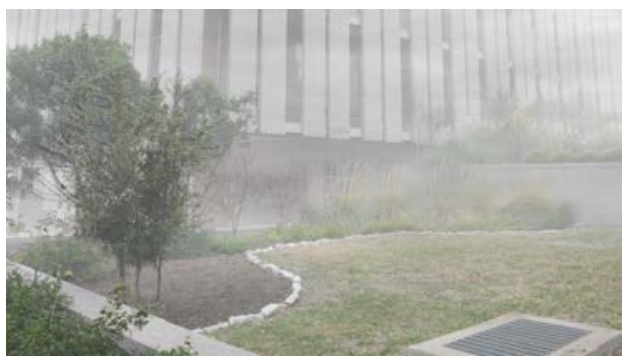

(c)

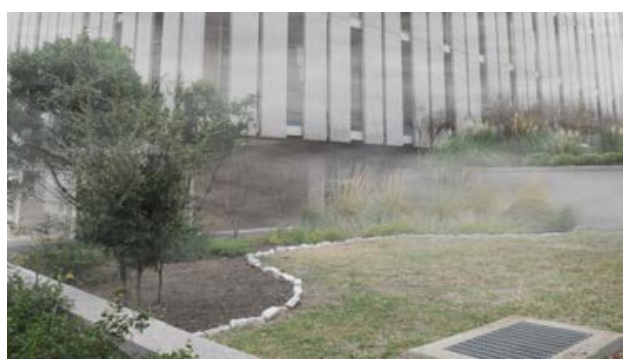

(e)

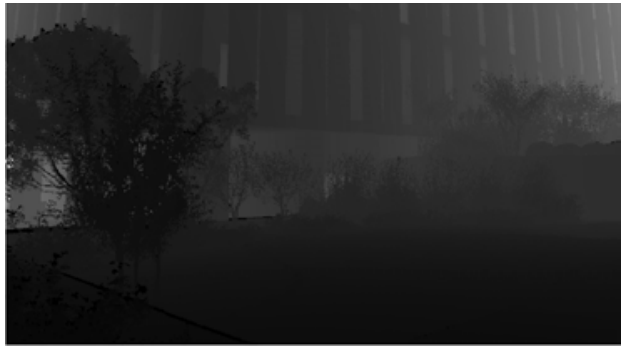

(b)

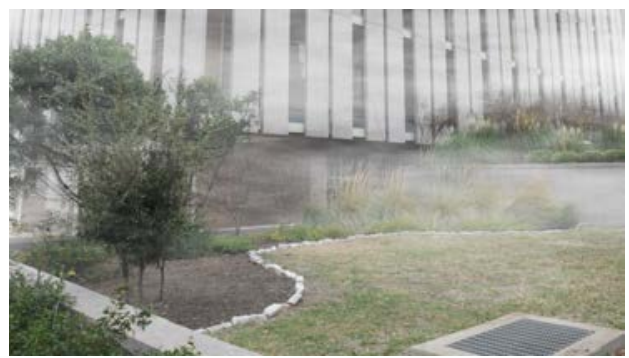

(d)

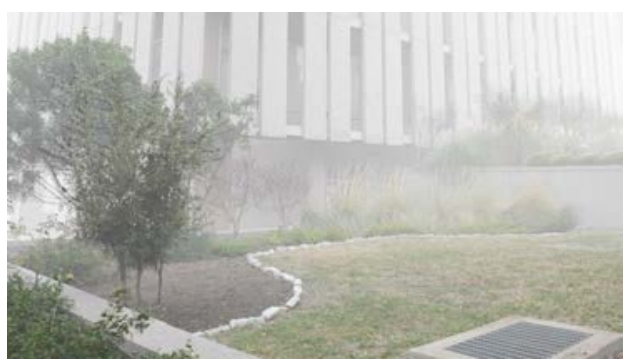

(f)

FIG. 4.13. Scene from the LIVE Color+3D Database. (a) Original scene. (b) Its corresponding range data (c)-(f) Input image with different synthetic heterogeneous fog added on it by following the method in 44 .

For measures based on the correlation, the considered measures are similar to the first two defined measures, by replacing the MSE measure by the correlation between the channels. Mathematically,

$\operatorname{Corr}_{\text {split }}=\sqrt{\text { Correlation }\left(I_{r}^{G T}, I_{r}^{m}\right)^{2}+\text { Correlation }\left(I_{g}^{G T}, I_{g}^{m}\right)^{2}+\operatorname{Correlation}\left(I_{b}^{G T}, I_{b}^{m}\right)^{2}}$,

and

$$
\text { Corr }_{l u m}=\text { Correlation }\left(I_{\text {lum }}^{G T}, I_{\text {lum }}^{m}\right),
$$

where Correlation gives us the correlation coefficient between the two images.

Results are shown in Table 4.1. Our EVID method outperforms all the others in the five different measures when the full set of images is considered (table rows labeled 'All'). When dividing the images depending on the fog model, EVID also 
TABLE 4.1

Quantitative results under the five different measures using the four different types of fog.

\begin{tabular}{|c|c|c|c|c|c|c|c|}
\hline Error measure & Fog model & 14 & 43 & 34 & 26 & 17 & EVID \\
\hline \multirow{5}{*}{$l_{2}-$ color } & het. air. & 4.8639 & 5.2898 & 6.824 & 6.3354 & 5.5232 & 4.8699 \\
\hline & het. air. and att. & 4.0625 & 3.7592 & 6.3964 & 5.9873 & 4.193 & 4.0129 \\
\hline & het. att. & 4.9991 & 4.4006 & 6.1187 & 6.2086 & 5.0711 & 4.4687 \\
\hline & homo. & 6.7278 & 7.2462 & 6.8091 & 6.9326 & 6.6519 & 5.857 \\
\hline & All & 5.1633 & 5.174 & 6.5371 & 6.366 & 5.3598 & 4.8021 \\
\hline \multirow{5}{*}{$M S E_{\text {lum }}$} & het. air. & 0.0249 & 0.0271 & 0.0484 & 0.0513 & 0.0355 & 0.023 \\
\hline & het. air. and att. & 0.0168 & 0.0135 & 0.0434 & 0.0494 & 0.0202 & 0.0162 \\
\hline & het. att. & 0.0269 & 0.0202 & 0.0394 & 0.0513 & 0.0305 & 0.0209 \\
\hline & homo. & 0.0519 & 0.0573 & 0.0481 & 0.0566 & 0.0532 & 0.0365 \\
\hline & All & 0.0301 & 0.0295 & 0.0448 & 0.0522 & 0.0349 & 0.0242 \\
\hline \multirow{5}{*}{$M S E_{\text {split }}$} & het. air. & 0.2691 & "0.281 & 0.3608 & 0.3328 & $\overline{0.3117}$ & 0.2582 \\
\hline & het. air. and att. & 0.221 & 0.1956 & 0.3391 & 0.3123 & 0.2379 & 0.2113 \\
\hline & het. att. & 0.2785 & 0.2393 & 0.3249 & 0.3276 & 0.2929 & 0.2377 \\
\hline & homo. & 0.3825 & 0.4042 & 0.3611 & 0.3701 & 0.3851 & 0.3191 \\
\hline & All & 0.2878 & 0.28 & 0.3465 & 0.3357 & 0.3069 & 0.2566 \\
\hline \multirow{5}{*}{ Corr $_{\text {split }}$} & het. air. & 1.2215 & 1.1914 & 0.738 & 0.9422 & 1.0925 & 1.2199 \\
\hline & het. air. att. & 1.442 & 1.3639 & 0.8806 & 1.0961 & 1.3315 & 1.4163 \\
\hline & het. att. & 1.2529 & 1.2573 & 0.9301 & 1.0125 & 1.2379 & 1.2884 \\
\hline & homo. & 1.028 & 1.045 & 0.8417 & 0.8959 & 1.0498 & 1.0765 \\
\hline & All & 1.2361 & 1.2144 & 0.8476 & 0.9867 & 1.1779 & 1.2503 \\
\hline \multirow{5}{*}{ Corr $_{l u m}$} & het. air. & 0.6958 & 0.6826 & 0.3937 & 0.5203 & 0.6144 & 0.6991 \\
\hline & het. air. att. & 0.8297 & 0.7839 & 0.4779 & 0.6299 & 0.7627 & 0.8180 \\
\hline & het. att. & 0.7147 & 0.7198 & 0.5036 & 0.5811 & 0.7062 & 0.7395 \\
\hline & homo. & 0.5598 & 0.5825 & 0.4547 & 0.4848 & 0.5742 & 0.5970 \\
\hline & All & 0.7 & 0.6922 & 0.4575 & 0.554 & 0.6644 & 0.7134 \\
\hline
\end{tabular}

outperforms the remaining ones for the homogeneous, heterogeneous attenuation and heterogeneous airlight fogs in most of the measures, being very close to the best method (Tarel and Hautiere method [43]) for the remaining fog types.

4.3. In-camera processing for physical-based approaches. Kochsmieder's law is physical and, therefore, it is directly linked with the information that reaches the camera sensor. Cameras, however, do not directly display this information due to camera limitations (such as glare 24, color gamut [28]) and camera processing options (white balance, type of scene). Even more, camera output is not in linear, but in gamma corrected form [46, 47.

Having said this, we want to point out that in the image dehazing literature the goal is obtaining visually pleasant images without the presence of haze or fog, i.e., when dehazing an image we do not aim to obtain the real color values of the scene, but a representation where we can observe the maximum information while having a visually pleasant look. And, to achieve this goal, the information given by the physical law (even if some corruption has been inserted by the in-camera processing) is shown to be essential. A clear example was given in section 4.1 .2 where our method is shown to dehaze the images in a better form than other contrast enhancement methods that 


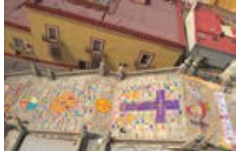

(a)

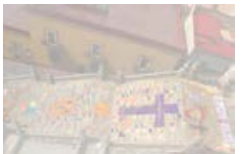

(b)

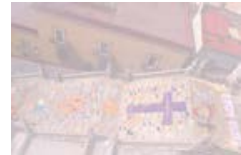

(c)

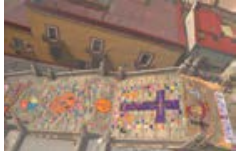

(d)

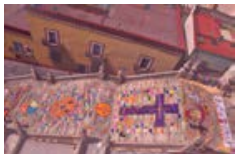

(e)

FIG. 4.14. Scene obtained from 47]. (a) Original scene. (b) Foggy-RAW image (c) Foggyprocessed image (d) Dehazing of Foggy-RAW image (e) Dehazing of Foggy-processed image

TABLE 4.2

Comparison of dehazing results pre- and post- camera processing.

\begin{tabular}{|c|c|c|c|c|}
\hline Error measure & Original RAW & RAW dehazed & Proc. dehazed & Deterioration \\
\hline$l_{2}-$ color & 12.3916 & 3.1547 & 3.4442 & $2.3365 \%$ \\
$M S E_{\text {lum }}$ & 0.1366 & 0.0083 & 0.0109 & $1.8944 \%$ \\
$M S E_{\text {split }}$ & 0.6333 & 0.1697 & 0.1913 & $3.4192 \%$ \\
Corr $_{\text {split }}$ & 1.4246 & 1.4358 & 1.4329 & $0.2021 \%$ \\
Corr $_{\text {lum }}$ & 0.8277 & 0.8467 & 0.8412 & $0.6617 \%$ \\
\hline
\end{tabular}

do not consider the physical information.

To test the impact of these factors in our results we performed a last experiment. We considered 8 non-camera processed, 12 bit RAW images from the dataset of [47]. (example image in Figure 4.14a)). For each of the images we computed a depth map 52 and used this map to obtain a homogeneous foggy-RAW image (Figure (4.14b)). We then applied to this foggy-RAW image the typical in-camera processing pipeline: we multiplied it by a $3 \times 3$ matrix transform that randomly deviates $\frac{1}{8}$ from the identity in each position to simulate the color compensation (white-balance, picture style), we applied a contrast enhancement in the form of a gamma function, and we added a channel-wise non-linearity to both the bright and dark pixels to emulate gamut mapping. Finally, we quantized the image to 8 bits. The result of this process can be seen in Figure (4.14c). Therefore, with this procedure we obtained a ground-truth image, a foggy non-camera processed image (foggy-RAW), and a foggy camera processed image (foggy-processed). Then, we dehazed the foggy-RAW and the foggy-processed images with our method and obtained the results presented in Figures 4.14d and 4.14e. We can see that qualitatively, the result after the camera processing steps is somewhat worse when compared to the ground-truth because it considers the color compensation matrix as part of the non-foggy original image, but, even in this case, it is still a very good solution to the dehazing problem. Quantitative results in the form of the mean for the 8 images are presented in Table 4.2. There, we compare the results of the original foggy image to the results of applying our method to both the foggy-RAW and the foggy-processed images. A last column is added to compute the percentage of deterioration in comparison to the foggy original image introduced by the in-camera processing. As it can be seen, this deterioration reaches its maximum at a $3.4197 \%$ for the $M S E_{\text {split }}$.

Finally, let us also remark that our approach considers Kochsmieder's law in a softer manner than the rest of the dehazing methods also based in this law. In particular, we employ this law to obtain an estimation of the mean of the clean image, but, once that mean has been estimated, we do not compute the transmission in order to dehaze the image; therefore, our method does not consider Kochsmieder's 
law for the computation of the final result.

5. Conclusions. We have proposed two substantial enhancements for the wellknown perceptual color correction framework of 3 in order to perform image dehazing and defogging. First, we have adapted the grey-world hypothesis to deal with hazy scenes. Second, we have included a set of terms to maximize inter-channels contrast, leading to the formulation of an Enhanced Variational Image Dehazing (EVID) method. Our numerical experiments demonstrate an excellent behavior of our EVID method, which is qualitatively comparable versus state-of-the-art methods for normally degraded images while outperforming other methods in more challenging scenarios. We have also shown how the proposed method quantitatively outperforms other state-of-the-art methods in various fog scenarios.

Even if the EVID method seems to be capable of handling uniform areas of a hazy image, such as sky regions, in an equal or better manner when compared to other recent algorithms, we have found that chromatic artifacts still appear sometimes under very large uniform areas. This limits the appeal of our approach to be applied, for instance, for artistic purposes. Further careful modifications of the energy in Eq. (3.10) may possibly solve this issue.

Appendix. Proof of Eq. (3.11). Let us denote each of the different terms appearing in the global energy in Eq. (3.10) as follows:

$$
\begin{aligned}
\mathrm{E}_{m v}\left(\mathrm{I}^{j}, \mu^{j}\right) & =\sum_{x}\left(\mathrm{I}^{j}(x)-\mu^{j}\right)^{2} \\
\mathrm{E}_{\mathrm{A} 2 \mathrm{D}}\left(\mathrm{I}^{j}\right) & =\sum_{x}\left(\mathrm{I}^{j}(x)-\mathrm{I}_{0}^{j}(x)\right)^{2} \\
\mathrm{E}_{\mathrm{C}}\left(\mathrm{I}^{j}, \mathrm{I}^{k}\right) & =\sum_{x, y} \omega(x, y)\left|\mathrm{I}^{j}(x)-\mathrm{I}^{k}(y)\right| .
\end{aligned}
$$

When $j=k$, Eq. A.3 represents the mono-channel contrast enhancement term, and when $j \neq k$, the inter-channel stretching term. With this notation, the full energy can be written as:

$$
E\left(\mathrm{I}^{j}\right)=\frac{\alpha}{2} \mathrm{E}_{m v}\left(\mathrm{I}^{j}, \mu^{j}\right)+\frac{\beta}{2} \mathrm{E}_{\mathrm{A} 2 \mathrm{D}}\left(\mathrm{I}^{j}\right)-\frac{\gamma}{2} \mathrm{E}_{\mathrm{C}}\left(\mathrm{I}^{j}, \mathrm{I}^{j}\right)-\frac{\eta}{2}\left(\mathrm{E}_{\mathrm{C}}\left(\mathrm{I}^{j}, \mathrm{I}^{j+1}\right)+\mathrm{E}_{\mathrm{C}}\left(\mathrm{I}^{j}, \mathrm{I}^{j+2}\right)\right) .
$$

Now, given a differentiable scalar function $\varphi$ and an arbitrary energy:

$$
\mathrm{E}(\mathrm{I})=\sum_{x} \varphi(\mathrm{I}(x))
$$

the chain rule states that its derivative in the $\delta \mathrm{I}$ direction is:

$$
\delta \mathrm{E}(\mathrm{I}, \delta \mathrm{I})=\sum_{x}\left(\left.\frac{\partial \varphi}{\partial I}\right|_{\mathrm{I}(x)} \cdot \delta \mathrm{I}(x)\right),
$$

which when applied to $\mathrm{E}_{m v}$ and $\mathrm{E}_{\mathrm{A} 2 \mathrm{D}}$ in Eqs. A.1 and A.2 yields:

$$
\begin{aligned}
\delta \mathrm{E}_{m v}\left(\left(\mathrm{I}^{j}, \mu^{j}\right), \delta \mathrm{I}^{j}\right) & =2 \sum_{x}\left(\mathrm{I}^{j}(x)-\mu^{j}\right) \delta \mathrm{I}^{j}(x), \\
\delta \mathrm{E}_{\mathrm{A} 2 \mathrm{D}}\left(\mathrm{I}^{j}, \delta \mathrm{I}^{j}\right) & =2 \sum_{x}\left(\mathrm{I}^{j}(x)-\mathrm{I}_{0}^{j}(x)\right) \delta \mathrm{I}^{j}(x) .
\end{aligned}
$$


To compute the variation of $\mathrm{E}_{\mathrm{C}}\left(\mathrm{I}^{j}, \mathrm{I}^{k}\right)$ in the direction of $\mathrm{I}^{j}$, we distinguish two cases. We assume first $j=k$, and approximate the non-differentiable absolute value by the function $z \mapsto|z|_{\epsilon}=\sqrt{z^{2}+\epsilon^{2}}$, the derivative of which is the $\operatorname{sign}_{\epsilon}$ function:

$$
\frac{d}{d z}|z|_{\epsilon}=\frac{z}{\sqrt{z^{2}+\epsilon^{2}}}
$$

that, as $\epsilon$ tends to 0 , approximates the sign function smoothly:

$$
\operatorname{sign}_{\epsilon}(z) \stackrel{\epsilon \rightarrow 0}{\longrightarrow} \operatorname{sign}(z)= \begin{cases}1 & \text { if } z>0 \\ 0 & \text { if } z=0 \\ -1 & \text { if } z<0 .\end{cases}
$$

Replacing the absolute value in Eq. A.3 by $|\cdot|_{\epsilon}$ we obtain a modified energy $\mathrm{E}_{\mathrm{C}}^{\epsilon}\left(\mathrm{I}^{j}, \mathrm{I}^{j}\right)$, the variation of which is given by:

$$
\begin{aligned}
\delta \mathrm{E}_{\mathrm{C}}^{\epsilon}\left(\left(\mathrm{I}^{j}, \mathrm{I}^{j}\right), \delta \mathrm{I}^{j}\right)= & \sum_{x}\left(\sum_{y} \omega(x, y) \operatorname{sign}_{\epsilon}\left(\mathrm{I}^{j}(x)-\mathrm{I}^{j}(y)\right)\right)\left(\delta \mathrm{I}^{j}(x)-\delta \mathrm{I}^{j}(y)\right) \\
= & \sum_{x} \sum_{y} \omega(x, y) \operatorname{sign}_{\epsilon}\left(\mathrm{I}^{j}(x)-\mathrm{I}^{j}(y)\right) \delta \mathrm{I}^{j}(x) \\
& -\sum_{x} \sum_{y} \omega(x, y) \operatorname{sign}_{\epsilon}\left(\mathrm{I}^{j}(x)-\mathrm{I}^{j}(y)\right) \delta \mathrm{I}^{j}(y) .
\end{aligned}
$$

Now, using the formula $\operatorname{sign}_{\epsilon}(z)=-\operatorname{sign}_{\epsilon}(-z)$, we rewrite the second term in the right hand side of the last equality:

$$
\begin{aligned}
\delta \mathrm{E}_{\mathrm{C}}^{\epsilon}\left(\mathrm{I}^{j}, \delta \mathrm{I}^{j}\right)= & \sum_{x} \sum_{y} \omega(x, y) \operatorname{sign}_{\epsilon}\left(\mathrm{I}^{j}(x)-\mathrm{I}^{j}(y)\right) \delta \mathrm{I}^{j}(x) \\
& +\sum_{x} \sum_{y} \omega(x, y) \operatorname{sign}_{\epsilon}\left(\mathrm{I}^{j}(y)-\mathrm{I}^{j}(x)\right) \delta \mathrm{I}^{j}(y) \\
= & \sum_{x} \sum_{y} \omega(x, y) \operatorname{sign}_{\epsilon}\left(\mathrm{I}^{j}(x)-\mathrm{I}^{j}(y)\right) \delta \mathrm{I}^{j}(x) \\
& +\sum_{y} \sum_{x} \omega(y, x) \operatorname{sign}_{\epsilon}\left(\mathrm{I}^{j}(x)-\mathrm{I}^{j}(y)\right) \delta \mathrm{I}^{j}(y) .
\end{aligned}
$$

In the above equality, we have interchanged the notation $x \leftrightarrow y$ in the last term. Since $\omega(x, y)$ is a symmetric function, and applying a discrete version of Fubini's Theorem, we can interchange the order of summation in the second term of the sum, and gather both terms arriving at:

$$
\delta \mathrm{E}_{\mathrm{C}}^{\epsilon}\left(\mathrm{I}^{j}, \delta \mathrm{I}^{j}\right)=2 \sum_{x}\left(\sum_{y} \omega(x, y) \operatorname{sign}_{\epsilon}\left(\mathrm{I}^{j}(x)-\mathrm{I}^{j}(y)\right) d y\right) \delta \mathrm{I}^{j}(x) .
$$

The variation of $\mathrm{E}_{\mathrm{C}}\left(\mathrm{I}^{j}, \mathrm{I}^{k}\right)$ in the direction of $\mathrm{I}^{j}$ when $j \neq k$ is a simplification of the above. Since in this case $\mathrm{I}^{k}$ can be considered a constant, we simply need to approximate the absolute value by $|\cdot|_{\epsilon}$ in order to directly apply the chain rule:

$$
\delta \mathrm{E}_{\mathrm{C}}^{\epsilon}\left(\left(\mathrm{I}^{j}, \mathrm{I}^{k}\right), \delta \mathrm{I}^{j}\right)=\sum_{x}\left(\sum_{y} \omega(x, y) \operatorname{sign}_{\epsilon}\left(\mathrm{I}^{j}(x)-\mathrm{I}^{k}(y)\right)\right) \delta \mathrm{I}^{j}(x) .
$$


By letting $\epsilon$ tend to zero in the Eqs. A.4 and A.5 and gathering together all variations, we can write the variation of the full energy in the direction of $\delta \mathrm{I}^{j}$ as:

$$
\begin{aligned}
\delta E\left(\mathrm{I}^{j}\right)= & \sum_{x}\left[\alpha\left(\mathrm{I}^{j}(x)-\mu^{j}\right)+\beta\left(\mathrm{I}^{j}(x)-\mathrm{I}_{0}^{j}(x)\right)-\gamma \sum_{y} \omega(x, y) \operatorname{sign}\left(\mathrm{I}^{j}(x)-\mathrm{I}^{j}(y)\right)\right. \\
& \left.-\eta\left(\sum_{y} \omega(x, y) \operatorname{sign}\left(\mathrm{I}^{j}(x)-\mathrm{I}^{j+1}(y)\right)+\sum_{y} \omega(x, y) \operatorname{sign}\left(\mathrm{I}^{j}(x)-\mathrm{I}^{j+2}(y)\right)\right)\right] \delta \mathrm{I}^{j}(x) .
\end{aligned}
$$

This shows that the gradient of the full energy amounts to the expression shown in Eq. (3.11).

\section{REFERENCES}

[1] C.O. Ancuti And C. Ancuti, Single Image Dehazing by Multi-Scale Fusion, IEEE Transactions on Image Processing, 22 (2013), pp. 3271-3282.

[2] M. Bertalmío, V. Caselles, and E. Provenzi, Issues About Retinex Theory and Contrast Enhancement, International Journal of Computer Vision, 83 (2009), pp. 101-119.

[3] M. Bertalmío, V. Caselles, E. Provenzi, and A. Rizzi, Perceptual color correction through variational techniques, IEEE transactions on image processing: a publication of the IEEE Signal Processing Society, 16 (2007), pp. 1058-1072.

[4] P. Carr and R. Hartley, Improved Single Image Dehazing Using Geometry, in Digital Image Computing: Techniques and Applications, 2009. DICTA '09., Dec. 2009, pp. 103-110.

[5] A. Chambolle and T. Pock, A First-Order Primal-Dual Algorithm for Convex Problems with Applications to Imaging, Journal of Mathematical Imaging and Vision, 40 (2011), pp. $120-145$.

[6] J.Y. Chiang and Y. Chen, Underwater Image Enhancement by Wavelength Compensation and Dehazing, IEEE Transactions on Image Processing, 21 (2012), pp. 1756-1769.

[7] F. Fang, F. Li, and T. Zeng, Single Image Dehazing and Denoising: A Fast Variational Approach, SIAM Journal on Imaging Sciences, 7 (2014), pp. 969-996.

[8] R. FATtal, Single Image Dehazing, in ACM SIGGRAPH 2008 Papers, SIGGRAPH '08, New York, NY, USA, 2008, ACM, pp. 72:1-72:9.

[9] — Dehazing using Color-Lines, in ACM Transaction on Graphics, New York, NY, USA, 2014, ACM.

[10] S. Ferradans, M. Bertalmío, E. Provenzi, and V. Caselles, An Analysis of Visual Adaptation and Contrast Perception for Tone Mapping, IEEE Transactions on Pattern Analysis and Machine Intelligence, 33 (2011), pp. 2002-2012.

[11] A. Galdran, D. Pardo, A. Picón, And A. Alvarez-Gila, Automatic Red-Channel underwater image restoration, Journal of Visual Communication and Image Representation, 26 (2015), pp. 132-145.

[12] A. Galdran, J. Vazquez-Corral, D. Pardo, and M. Bertalmío, A Variational Framework for Single Image Dehazing, in Proc. of the 4th Color and Photometry in Computer Vision Workshop (ECCV 2014), Zurich, Sept. 2014.

[13] Pascal Getreuer, Automatic Color Enhancement (ACE) and its Fast Implementation, Image Processing On Line, 2 (2012), pp. 266-277.

[14] K.B. Gibson And T.Q. NGuYen, Fast single image fog removal using the adaptive Wiener filter, in 2013 20th IEEE International Conference on Image Processing (ICIP), Sept. 2013, pp. $714-718$.

[15] N. Hautiere, J. P Tarel, and D. Aubert, Towards Fog-Free In-Vehicle Vision Systems through Contrast Restoration, in IEEE Conference on Computer Vision and Pattern Recognition, 2007. CVPR '07, June 2007, pp. 1-8.

[16] K. He, J. Sun, And X. TAng, Single Image Haze Removal Using Dark Channel Prior, in IEEE Conference on Computer Vision and Pattern Recognition, 2009. CVPR 2009, June 2009, pp. $1956-1963$.

[17] - Single Image Haze Removal Using Dark Channel Prior, IEEE Transactions on Pattern Analysis and Machine Intelligence, 33 (2011), pp. 2341-2353.

[18] R. Kaftory, Y.Y. Schechner, And Y.Y. ZeEvi, Variational Distance-Dependent Image 
Restoration, in IEEE Conference on Computer Vision and Pattern Recognition, 2007. CVPR '07, 2007, pp. 1-8.

[19] J. Kopf, B. Neubert, B. Chen, M. Cohen, D. Cohen-Or, O. Deussen, M. Uyttendaele, And D. Lischinski, Deep Photo: Model-based Photograph Enhancement and Viewing, in ACM SIGGRAPH Asia 2008 Papers, SIGGRAPH Asia '08, New York, NY, USA, 2008, ACM, pp. 116:1-116:10.

[20] H. Koschmieder, Theorie der horizontalen Sichtweite: Kontrast und Sichtweite, Keim \& Nemnich, 1925.

[21] Y. Lai, Y. Chen, C. Chiou, and C. Hsu, Single-Image Dehazing via Optimal Transmission Map Under Scene Priors, IEEE Transactions on Circuits and Systems for Video Technology, 25 (2015), pp. 1-14.

[22] J. Long, Z. Shi, W. Tang, and C. Zhang, Single Remote Sensing Image Dehazing, IEEE Geoscience and Remote Sensing Letters, 11 (2014), pp. 59-63.

[23] A. Makarau, R. Richter, R. Muller, and P. Reinartz, Haze Detection and Removal in Remotely Sensed Multispectral Imagery, IEEE Transactions on Geoscience and Remote Sensing, 52 (2014), pp. 5895-5905.

[24] J. J. MCCANn AND A. RIzZI, Veiling glare: the dynamic range limit of HDR images, vol. 6492, 2007, pp. 649213-649213-10.

[25] E. J. McCartney, Optics of the Atmosphere: Scattering by Molecules and Particles, Wiley, Jan. 1976.

[26] G. Meng, Y. Wang, J. Duan, S. Xiang, and C. Pan, Efficient Image Dehazing with Boundary Constraint and Contextual Regularization, in 2013 IEEE International Conference on Computer Vision (ICCV), Dec. 2013, pp. 617-624.

[27] W. E. K. Middleton, Vision through the atmosphere, University of Toronto Press, 1952.

[28] J. Morovič, Color Gamut Mapping, Wiley, Chichester, England ; Hoboken, NJ, 1 edition ed., July 2008.

[29] S.G. NARASimhan And S.K. NAYAR, Chromatic framework for vision in bad weather, in IEEE Conference on Computer Vision and Pattern Recognition, 2000. Proceedings, vol. 1, 2000, pp. 598-605 vol.1.

[30] - Vision and the Atmosphere, International Journal of Computer Vision, 48 (2002), pp. 233-254.

[31] - Contrast restoration of weather degraded images, IEEE Transactions on Pattern Analysis and Machine Intelligence, 25 (2003), pp. 713-724.

[32] — Interactive (de) weathering of an image using physical models, IEEE Workshop on Color and Photometric Methods in Computer Vision, 6 (2003), p. 1.

[33] S.K. Nayar and S.G. Narasimhan, Vision in bad weather, in The Proceedings of the Seventh IEEE International Conference on Computer Vision, 1999, vol. 2, 1999, pp. 820-827 vol.2.

[34] K. Nishino, L. Kratz, and S. Lombardi, Bayesian Defogging, International Journal of Computer Vision, 98 (2012), pp. 263-278.

[35] R. Palma-Amestoy, E. Provenzi, M. Bertalmio, and V. Caselles, A Perceptually Inspired Variational Framework for Color Enhancement, IEEE Transactions on Pattern Analysis and Machine Intelligence, 31 (2009), pp. 458-474.

[36] A. Rizzi, C. Gatta, and D. Marini, A New Algorithm for Unsupervised Global and Local Color Correction, Pattern Recogn. Lett., 24 (2003), pp. 1663-1677.

[37] G. Sapiro and V. Caselles, Histogram Modification via Differential Equations, Journal of Differential Equations, 135 (1997), pp. 238-268.

[38] L. Schaul, C. Fredembach, and S. Susstrunk, Color image dehazing using the near-infrared, in 2009 16th IEEE International Conference on Image Processing (ICIP), Nov. 2009, pp. $1629-1632$.

[39] Y.Y. Schechner, S.G. Narasimhan, And S.K. Nayar, Instant dehazing of images using polarization, in Proceedings of the 2001 IEEE Computer Society Conference on Computer Vision and Pattern Recognition, 2001. CVPR 2001, vol. 1, 2001, pp. I-325-I-332 vol.1.

[40] Y. Shiau, H. Yang, P. Chen, and Y. Chuang, Hardware Implementation of a Fast and Efficient Haze Removal Method, IEEE Transactions on Circuits and Systems for Video Technology, 23 (2013), pp. 1369-1374.

[41] C. Su, L.K. Cormack, And A.C. Bovik, Color and Depth Priors in Natural Images, IEEE Transactions on Image Processing, 22 (2013), pp. 2259-2274.

[42] R.T. TAN, Visibility in bad weather from a single image, in IEEE Conference on Computer Vision and Pattern Recognition, 2008. CVPR 2008, June 2008, pp. 1-8.

[43] J. P TAREL AND N. HaUtiere, Fast visibility restoration from a single color or gray level image, in 2009 IEEE 12th International Conference on Computer Vision, Sept. 2009, pp. 22012208. 
[44] J.-P. Tarel, N. Hautiere, L. Caraffa, A. Cord, H. Halmaoui, and D. Gruyer, Vision Enhancement in Homogeneous and Heterogeneous Fog, IEEE Intelligent Transportation Systems Magazine, 4 (2012), pp. 6-20.

[45] J.-P. Tarel, N. Hautiere, A. Cord, D. Gruyer, and H. Halmaoui, Improved visibility of road scene images under heterogeneous fog, in 2010 IEEE Intelligent Vehicles Symposium (IV), June 2010, pp. 478-485.

[46] J. Vazquez-Corral and M. Bertalmío, Color stabilization along time and across shots of the same scene, for one or several cameras of unknown specifications, IEEE transactions on image processing: a publication of the IEEE Signal Processing Society, 23 (2014), pp. $4564-4575$

[47] - Simultaneous Blind Gamma Estimation, IEEE Signal Processing Letters, 22 (2015), pp. $1316-1320$

[48] Y. Wang And C. FAn, Single Image Defogging by Multiscale Depth Fusion, IEEE Transactions on Image Processing, 23 (2014), pp. 4826-4837.

[49] I. Yoon, S. Kim, D. Kim, M.H. HAYES, AND J. PAIK, Adaptive defogging with color correction in the HSV color space for consumer surveillance system, IEEE Transactions on Consumer Electronics, 58 (2012), pp. 111-116.

[50] S.W. Zamir, J. VazQuez-Corral, and M. Bertalmío, Gamut Mapping in Cinematography Through Perceptually-Based Contrast Modification, IEEE Journal of Selected Topics in Signal Processing, 8 (2014), pp. 490-503.

[51] Y. Zhang AND B. Guindon, Quantitative assessment of a haze suppression methodology for satellite imagery: effect on land cover classification performance, IEEE Transactions on Geoscience and Remote Sensing, 41 (2003), pp. 1082-1089.

[52] S. Zhuo And T. Sim, Defocus map estimation from a single image, Pattern Recogn., 44 (2011), pp. $1852-1858$ 